Article

\title{
Chelation-Assisted Substrate-Controlled Asymmetric Lithiation-Allylboration of Chiral Carbamate 1,2,4-Butanetriol Acetonide
}

\author{
Adeem Mahmood ${ }^{1,2, *}$, Hamad Z. Alkhathlan ${ }^{1, *}$, Saima Parvez ${ }^{3}$, Merajuddin Khan ${ }^{1}$ \\ and Sohail A. Shahzad ${ }^{2}$
}

1 Department of Chemistry, College of Science, King Saud University, Riyadh 11451, Saudi Arabia; E-Mail: mdk.chem@gmail.com

2 Department of Chemistry, COMSATS Institute of Information Technology, Abbottabad 22060, Pakistan; E-Mail: sohail_chem@yahoo.com

3 School of Medicine, Shandong University, Jinan 250012, China; E-Mail: daring_saima@hotmail.com

* Authors to whom correspondence should be addressed; E-Mails: dr.adeem@hotmail.com (A.M.); khathlan@ksu.edu.sa (H.Z.A.); Tel.: +966-469-9638 (A.M.); +966-467-5896 (H.Z.A.); Fax: +966-467-5992 (H.Z.A).

Academic Editor: Jean Jacques Vanden Eynde

Received: 5 April 2015 / Accepted: 20 May 2015 /Published: 28 May 2015

\begin{abstract}
The lithiation of 2-(2,2-dimethyl-1,3-dioxolan-4-yl)ethyl diisopropylcarbamate (1) is achieved freely by sec-butyllithium in diethylether with high $l k$-diastereoselectivity: the bicyclic chelate complexes $\mathbf{3 a}$ and $\mathbf{3 b}$ are reacted with electrophiles to form optically active precursors $\mathbf{4 a}$ and $\mathbf{4 b}$ with $>95 \%$ diastereoselectivity. In addition, tertiary diamines can undergo an external complexation in contest with the internal oxygen ligand, leading to improved stereoselectivities. The further reactions of lithiated carbamates with trans alkenyl-9-BBN derivatives after 1,2 metallate rearrangements, gave the key intermediate $\alpha$-substituted allylic boranes 7 . Subsequent allylboration of aldehydes gave (Z)-anti-homoallylic alcohols 8 in good yield and excellent $d . r$.
\end{abstract}

Keywords: lithiation; borylation; allylation; chelation 


\section{Introduction}

Hoppe and co-workers investigated the lithiation of carbamates derived from non-racemic chiral primary alcohols generating organolithium intermediates which undergo electrophile-dependent stereodivergent substitution that often have remarkable configurational stability [1-4]. This stability is due to dipole stabilization and intramolecular chelation of the lithium counterion by the carbamoyl group [5]. The importance of carbamate group, in the enhancement of the kinetic acidity of $\alpha$-protons and the stabilization of lithio derivatives by chelation through one of the oxygen lone-pairs has been recognized by many research groups [1]. It has been shown that the sterically congested 1,2,4-butanetriol acetonide carbamate could be lithiated by $s$-BuLi/TMEDA in diethylether to form an $\alpha$-lithiated species [1,2], whilst in the presence of the chiral diamine (-)-sparteine, pro- $S \mathrm{H}$ deprotonates preferably and the configurationally stable lithiated complexes are subsequently trapped with different electrophiles with retention of configuration [6]. Generally, it has been considered that the remote donor substituents or groups, such as acetonide group, could also interfere in lithiation [7,8].

Aggarwal and co-workers have investigated the chelation-assisted substrate-controlled (CASC), asymmetric lithiation, and allylboration through less-sterically demanding chiral carbamates and explored its application for selectively making C-C bonds [9-18]. Previously, we have described this methodology to obtain highly selective products through substituted boranes (trans-alkenyl-9-BBN) and boronic esters and their reactions with sparteine-complexed lithiated carbamates [19]. Moreover, this protocol was further applied in the formation of penta-substituted tetrahydropyrans through the Prins cyclization [20].

\section{Results and Discussion}

In this paper, we optimized the use of the important enantioenriched stereodirecting carbamate (S)-2-(2,2-dimethyl-1,3-dioxolan-4-yl)ethyl diisopropylcarbamate (1) in lithiation, electrophilic addition, borylation and allylation reactions, involving two different protocols e.g., with/without the addition of an external bi-dentate diamine, such as TMEDA/(-)-sparteine/(+)-sparteine surrogate. This carbamate $\mathbf{1}$ is only differs in the leaving group moiety i.e., an open chain OCb instead of the cyclic OCby, from Hoppe's carbamate used nearly 20 years back [3].

\subsection{Chemistry}

Two procedures were adopted for the deprotonation of the carbamate 1, one in the presence of external ligands (procedure A) and another with no external ligands involved (procedure B) (Scheme 1). Although very similar results were obtained as per Hoppe [3], but products more bench stable (no isomerization occured even at longer time and elevated temperatures), and reactions are quicker, and give high yields and good d.r. This shows that the expulsion of an unhindered carbamate leaving group $(\mathrm{OCb})$ could be quicker than that of a bulky carbamate (OCby). The protocol involved the ChelationDirected-Asymmetric-Lithiation (CDAL) of 1,2,4-butanetriol acetonide by the drop-wise addition of $s \mathrm{BuLi}$ in $\mathrm{Et}_{2} \mathrm{O}$ or $s \mathrm{BuLi}$ in $\mathrm{Et}_{2} \mathrm{O} /$ additative chelating ligands e.g., $N, N, N, N$-tetramethylethylenediamine (TMEDA), (-)-sparteine or (+)-sparteine surrogate. An effective substrate-inherent chiral induction was exploited with acetonide-type carbamate 1. Here we report on the generation of chiral synthetic 
equivalents for the 1,2,4-trihydroxybutanide ion 1a, showing the stereo-directing influence of the protected 3-hydroxy group. Furthermore the conformational strain of the dioxalane ring is noteworthy for attaining good diastereoselectivities.

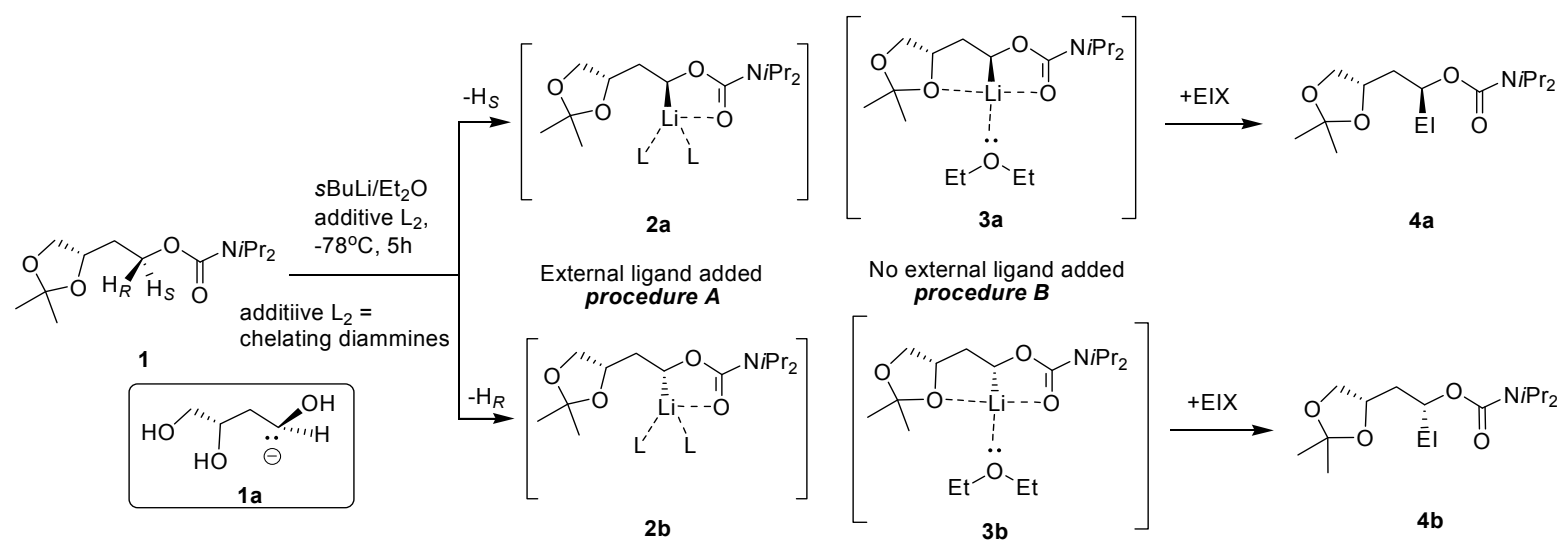

Scheme 1. Chelation-directed lithiation/ES of carbamate 1.

The relative rate for the deprotonation of the diastereotopic protons (pro-S or pro- $R$ ) reflects the rate-ratio in the presence of chiral inductor (diamine additives). The configurationally stable chiral ion pairs or lithio-intermediate 2a originates preferably when we used (-)-sparteine as an external chiral bi-dentate ligand, following the abstraction of the pro-S proton. Subsequent trapping of this configured complex 2a with different electrophiles (with retention of configuration) would then furnish $\mathbf{4 a}$ in good yield and high d.r. (Table 1).

Table 1. The chelation-directed asymmetric lithiation of carbamate $\mathbf{1}$.

\begin{tabular}{|c|c|c|c|c|c|}
\hline Entry & EIX & Additive $L_{2}$ & Yield ${ }^{\mathrm{a}}(\%)$ & d.r. ${ }^{\mathrm{b}} 4 \mathrm{a}: 4 \mathrm{~b}$ & $[\alpha]_{D}^{23}$ (conc., $\left.\mathrm{CH}_{3} \mathrm{OH}\right)$ \\
\hline \multirow{4}{*}{ I } & \multirow{4}{*}{$\mathrm{PhCHO}$} & TMEDA & 69 & $51: 49$ & $\mathrm{nd}^{\mathrm{c}}$ \\
\hline & & $(-)-\mathrm{Sp}$ & 74 & $98: 2$ & $-17.4(1.0)$ \\
\hline & & $(+)$-Sp surrogate & 53 & $20: 80$ & nd \\
\hline & & None & 84 & $98: 2$ & $-17.2(1.0)$ \\
\hline \multirow{4}{*}{ II } & \multirow{4}{*}{$\mathrm{Me}_{3} \mathrm{SnCl}$} & TMEDA & 80 & $53: 43$ & nd \\
\hline & & $(-)-S p$ & 75 & $97: 3$ & $+13.0(1.2)$ \\
\hline & & $(+)$-Sp surrogate & nd & nd & nd \\
\hline & & None & 78 & $98: 2$ & $+11.3(1.1)$ \\
\hline \multirow{4}{*}{ III } & \multirow{4}{*}{$\mathrm{PhCOPh}$} & TMEDA & 72 & $50: 50$ & nd \\
\hline & & $(-)-S p$ & 80 & $95: 5$ & $+23.6(1.0)$ \\
\hline & & $(+)$-Sp surrogate & 61 & 29:71 & nd \\
\hline & & None & 84 & $95: 5$ & $+27.3(1.5)$ \\
\hline
\end{tabular}

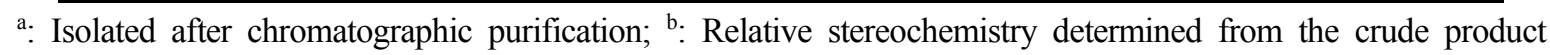
${ }^{1} \mathrm{H}-\mathrm{NMR}$ spectra; ${ }^{\mathrm{c}}$ : Not determined. 
Indeed, the opposite diastereomer $\mathbf{4 b}$ to $(-)$-sparteine can be effectively achieved through the appropriate choice of chiral diamines employed ((-)-sparteine, or (+)-sparteine surrogate) [21-23] (procedure $A$ ), whilst the racemic mixture with 1:1 ratio of the diastereomeric complexes $\mathbf{2 a}$ and $\mathbf{2 b}$ arises with sec-butyllithium/TMEDA. Interestingly, the diastereomeric ratio in $\mathbf{4 a}$ and $\mathbf{4 b}$ adequately increased to 98:2 when the lithiation was done without the addition of any external chelating diamine (procedure B).

From these results, it is clearly indicated that the intra- and intermolecular complexation involved an almost similar rate in the kinetically controlled deprotonation of the diastereotopic $\mathrm{H}_{S}$ and $\mathrm{H}_{R}$ protons in the carbamate ester. If no external bidentate ligands are linked to it then the $\beta$-oxygen atom acts mainly as a ligand to the lithium cation providing the bicyclic chelate complexes $\mathbf{3 a}$ or $\mathbf{3 b}$, where two neighboring five-membered rings are trans-annulated to the central ring, in contrast to the intermediates $\mathbf{2 a}$ or $\mathbf{2 b}$ which are stabilized through intermolecular complexation with an external bidenate ligand e.g., TMEDA or $(-)$-sparteine or $(+)$-sparteine surrogate. It is noteworthy that chiral induction [24] arises in the deprotonation step due to the presence of a good donor substituent next to a stereogenic $\mathrm{C}$ atom in the $\gamma$ - or $\delta$-position and therefore high substrate-controlled diastereoselectivities can be easily achieved. A bicyclic chelate complex of the type $\mathbf{3 a}$ is generated in the presence of $(-)$-sparteine, even if the specific stereochemistry of the bis-chelate complexes like $\mathbf{2 a}, \mathbf{2} \mathbf{b}$ or $\mathbf{3 a}, \mathbf{3 b}$ is doubtful. It is likely that a seven- or eight-membered ring might form due to the fixing of the more effective coordinating carbonyl group of the $\gamma$ - or $\delta$-carbamoyloxy residue. In addition, $\mathrm{Et}_{2} \mathrm{O}$ coordinates in monodentate fashion and hence the more favorable exo-position of the electrophile determines the transition state. It is believed that the formation of the anti-annulated tricyclic chelate complex 3a is highly selective despite the fact that the lithiation step is kinetically controlled, whilst, in the presence of external ligands e.g., (-)-sparteine, due to the mismatched pair situation; the connection towards the abstraction of the pro-S proton is further enhanced, because in this case $\mathbf{3 b}$ is no longer evident in the reaction mixture. The 2,2-dimethyl-substituted 1,3-dioxolane ring in the carbamate $\mathbf{1}$ is simply a weak ligand for lithiation, and as with sparteine, it is expelled by TMEDA. As Table 1 shows, diastereoselectivity is reduced under these conditions and is reversed by means of $(+)$-sparteine surrogate due to preference for the pro- $R$ proton [25]. Furthermore the normal deprotonation pathway relates to the presence of a bidentate complexing ligand where intramolecular complexation is not concerned. Also the ion pair 3a reacts efficiently with electrophiles and consequently the substrate generates a valuable synthetic equivalent to the $(S)$-1,3,4-trihydroxybutanide 1a [26,27].

The second part of this paper illustrates the allylboration of aldehydes to form $\mathrm{C}-\mathrm{C}$ bonds with control over relative and absolute stereochemistry (Table 2) [28-40]. Thus a wide scope and convergent method has been introduced for reacting lithiated carbamates with vinylboranes/boronic esters and aldehydes to give 1,2,4-trisubstituted homoallylic alcohols with full stereo control [19]. It has been described that by applying $\alpha$-substituted allyl boranes, it is possible to control the initial double bond geometry and all three element of stereochemistry of the homoallylic alcohol products (enantioselectivity, $E / Z$-stereochemistry and syn/anti stereochemistry) without any additional stereodirecting reagents.

The present strategy involves the reaction of lithiated carbamate 2 with alkyl substituted $B$-vinyl-(9-BBN) 5 to prepare potentially the key intermediate $\alpha$-substituted allylborane 7 through 1,2-metallate rearrangement product $\mathbf{6}$. The subsequent allylation reaction with different choices of aldehydes (e.g., PhCHO, CyhexCHO and $n \mathrm{BuCHO}$ ) gave exclusively the anti-(Z)-homoallylicsec-alcohols 8. 
No isomerization of the labile $\alpha$-substituted allylic borane 7 during the 1,2-metalate rearrangement was observed. This could be achieved by simply adding the aldehyde to the ate-complex $\mathbf{6}$ at low temperature after 1,2-metalate and rearrangement would occur upon warming to give the allyl borane 7 which would subsequently undergo allylation to give $\mathbf{8}$ before isomerization could occur. This protocol was found to be successful with a range of representative trans-vinylboranes $\mathbf{5 a}$ and $\mathbf{5 b}$, carbamate $\mathbf{1}$, and aldehydes (Table 2). In all cases, good diastereomeric ratios with moderate yields were observed.

Table 2. Synthesis of anti-(Z)-homoallylic alcohols through the lithiation/allylboration method.

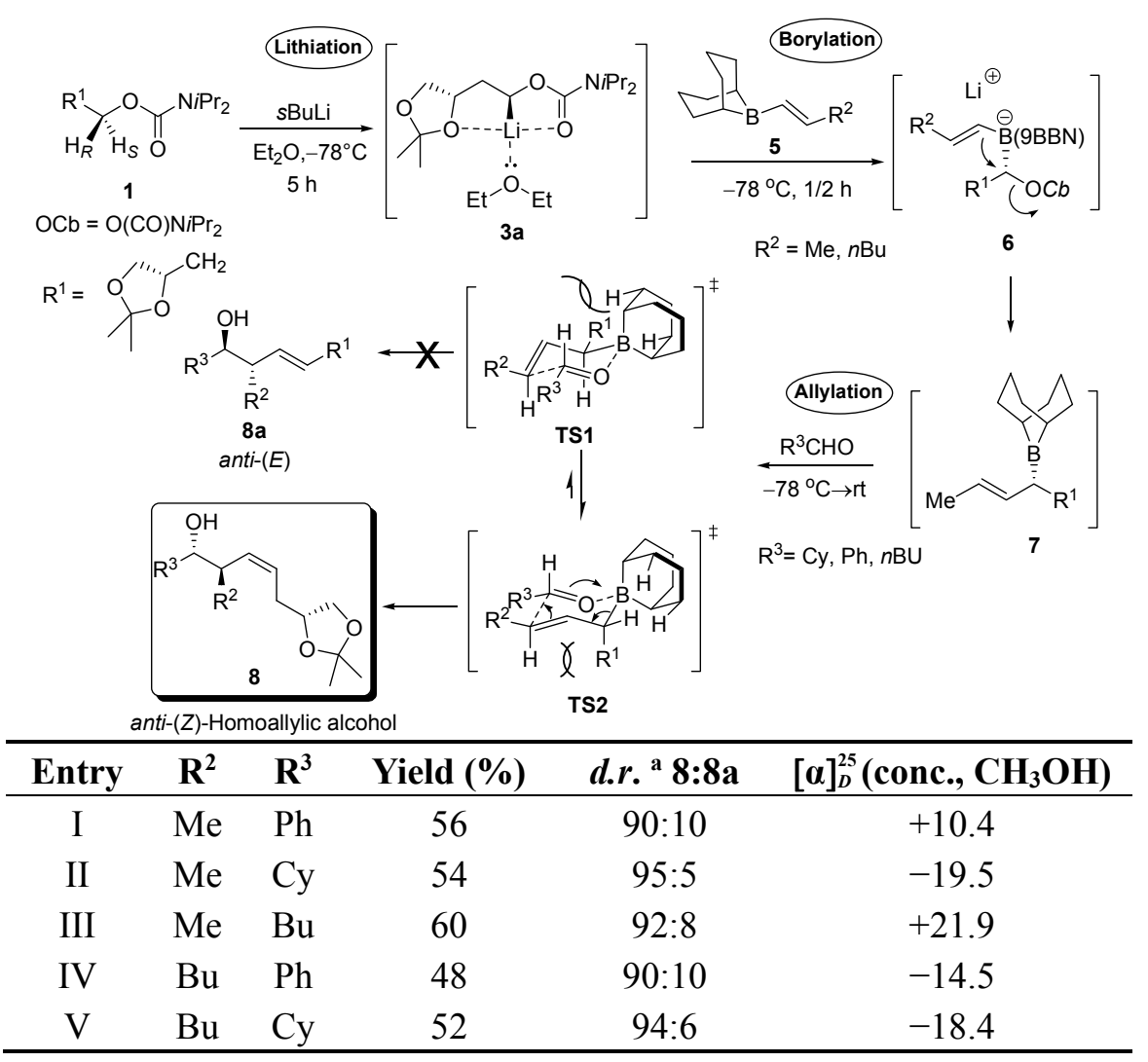

a: Relative Stereochemistry determined by the crude product ${ }^{1} \mathrm{H}-\mathrm{NMR}$-spectra.

The high selectivity originates from the closed 6-membered chair transition-state structures involved in minimizing non-bonded steric interactions [19,41], which can be rationalized by the increased steric hindrance in the transition-state structure TS2 compared to TS1 [42]. Severe steric hindrance between the 9-BBN ring and $\mathrm{R}^{1}$ would push the $\alpha$-carbon substituent into a pseudo-axial position resulting in the anti-diastereoisomer and (Z)-alkene geometry [43-46]. It is interesting to note that external complexation of tertiary diamines can compete with the internal oxygen ligand, furnishing stereoselectivities with good diastereoselectivities in the desired compounds.

\section{Experimental Section}

\subsection{General Information}

All air- and water-sensitive reactions were carried out in oven-dried $\left(180{ }^{\circ} \mathrm{C}\right)$ glassware and under an Air atmosphere using standard Schlenk techniques. Anhydrous solvents were prepared using a Grubbs-type anhydrous solvent drying columns. ${ }^{1} \mathrm{H}$ - and ${ }^{13} \mathrm{C}-\mathrm{Nuclear}$ Magnetic Resonance (NMR) 
spectra were acquired at various field strengths as indicated, and were referenced to $\mathrm{CHCl}_{3}(7.27$ and $77.0 \mathrm{ppm}$ for ${ }^{1} \mathrm{H}$ and ${ }^{13} \mathrm{C}$, respectively) or TMS (0.00 ppm for ${ }^{1} \mathrm{H}$ and $\left.{ }^{13} \mathrm{C}\right) .{ }^{1} \mathrm{H}$ - and ${ }^{13} \mathrm{C}-\mathrm{NMR}$ spectra are shown in the Supplementary Materials. ${ }^{11} \mathrm{~B}-\mathrm{NMR}$ spectra were recorded with complete proton decoupling using $\mathrm{BF}_{3} \cdot \mathrm{Et}_{2} \mathrm{O}(0.00 \mathrm{ppm})$ as an external standard. Assignment of signals in ${ }^{1} \mathrm{H}$ - and ${ }^{13} \mathrm{C}$-spectra was performed using ${ }^{1} \mathrm{H}-{ }^{1} \mathrm{H}$ COSY, DEPT, HMQC and HMBC, where appropriate. Low- and high-resolution mass spectra were recorded using Electron Impact (EI), Chemical Ionization (CI) or Electron-Spray Ionization (ESI) techniques. For $\mathrm{CI}$, methane or $\mathrm{NH}_{4} \mathrm{OAc} / \mathrm{MeOH}$ were used. Analytical TLC: aluminum backed plates pre-coated $(0.25 \mathrm{~mm})$ with Silica Gel 60 F254 (Merck Millipore, Darmstadt, Germany). Compounds were visualized by exposure to UV-light or by staining with $5 \%$ solution of $\left(\mathrm{NH}_{4}\right)_{2} \mathrm{Mo}_{7} \mathrm{O}_{24} 4 \mathrm{H}_{2} \mathrm{O}$ in EtOH followed by heating. Flash chromatography was carried out using Merck silica Gel 60, 0.040-0.063 mm particle size. Melting points were determined with a Boetius hot stage apparatus and were not corrected. All IR data were obtained on a Perkin-Elmer Spectrum One FT-IR spectrometer (Perkin-Elmer, Boston, MA, USA). N,N,N,N-Tetramethylethylenediamine (TMEDA) was purchased from Sigma-Aldrich (Gillingham, UK) and (-)-sparteine was purchased from Aldrich (5 years back from Gillingham, UK). $(+)$-sparteine surrogate was synthesized from commercially available (-)-cytisine [21]. Both were distilled under reduced pressure over $\mathrm{CaH}_{2}$ prior to use. 9-((E)-Prop1-enyl)-9-borabicyclo[3.3.1]nonane (9-BBN) as dimer was purchased from Aldrich. $s \mathrm{BuLi}$ (1.3 M solution in cyclohexane/hexanes, 92:8) was purchased from Sigma-Aldrich.

\section{2. (S)-2-(2,2-Dimethyl-1,3-dioxolan-4-yl)ethyl Diisopropylcarbamate (1)}

To a solution of $N, N$-diisopropylcarbamyl chloride $(8.80 \mathrm{~g}, 55.0 \mathrm{mmol})$ and $\mathrm{NEt}_{3}(8.15 \mathrm{~mL}, 58.0 \mathrm{mmol})$ in $\mathrm{CH}_{2} \mathrm{Cl}_{2}(100 \mathrm{~mL})$ was added $(S)$-3-acetonide-ethyl-1-ol $(7.60 \mathrm{~mL}, 55.0 \mathrm{mmol})$. This mixture was then heated to reflux and stirred for $24 \mathrm{~h}$. The reaction was cooled to rt. and $\mathrm{H}_{2} \mathrm{O}(100 \mathrm{~mL})$ was added. The aqueous layer was extracted with $\mathrm{CH}_{2} \mathrm{Cl}_{2}(3 \times 75 \mathrm{~mL})$ and the combined organic layers were dried $\left(\mathrm{MgSO}_{4}\right)$ and concentrated in vacuo to give the crude product as an orange oil. The crude oil was purified by flash chromatography (10\% EtOAc/petroleum ether) to yield $\mathbf{1}$ as a colourless oil $(6.64 \mathrm{~g}$, $54 \%) . R_{\mathrm{f}}=0.34\left(15 \%\right.$ EtOAc in petroleum ether); $[\alpha]_{D}^{24}-24.3\left(c 0.7, \mathrm{CH}_{2} \mathrm{Cl}_{2}\right) ; \mathrm{IR} v_{\max }$ (neat) $/ \mathrm{cm}^{-1} 2972$, 1690, 1442, 1312, 1218, 1069; ${ }^{1} \mathrm{H}-\mathrm{NMR}\left(\mathrm{CDCl}_{3}, 270 \mathrm{MHz}\right) ; \delta: 4.21$ (2 H, d, J=5.7 Hz, CH2 $\left.\mathrm{OC}\right), 4.08$ $\left(2 \mathrm{H}, \mathrm{t}, J=6.1 \mathrm{~Hz}, \mathrm{CH}_{2} \mathrm{CH}_{2} \mathrm{O}\right), 3.98-3.61\left(2 \mathrm{H}\right.$, br. m, $2 \times \mathrm{CH}$ ), 3.58-3.52 (1 H, m, $\left.\mathrm{CH}_{2} \mathrm{CHO}\right), 1.92-1.89$ $\left(2 \mathrm{H}, \mathrm{m}, \mathrm{CHCH}_{2} \mathrm{CH}_{2}\right), 1.37(3 \mathrm{H}, \mathrm{s}, \mathrm{CH} 3), 1.31(3 \mathrm{H}, \mathrm{s}, \mathrm{CH}), 2.00\left(12 \mathrm{H}, \mathrm{d}, J=6.8 \mathrm{~Hz}, 4 \times \mathrm{CH}_{3}\right)$;

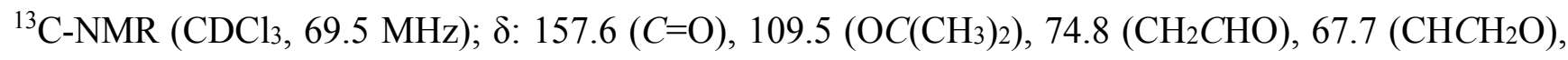
$57.0\left(\mathrm{CH}_{2} \mathrm{O}\right), 54.8(2 \times \mathrm{CH}), 31.5\left(\mathrm{CH}_{2}\right), 26.8\left(2 \times \mathrm{CH}_{3}\right), 21.2\left(4 \times \mathrm{CH}_{3}\right) ; \mathrm{HRMS}(\mathrm{ESI})$ calcd. For $\mathrm{C}_{14} \mathrm{H}_{27} \mathrm{NO}_{4} \mathrm{Na}$ : 274.2011; Found: 274.2018; Anal. Calcd for $\mathrm{C}_{14} \mathrm{H}_{27} \mathrm{NO}_{4}$ : C, 61.51; H, 9.96; N, 5.12; Found: C, 61.48; H, 9.98; N, 5.09.

\subsection{9-((E)-Prop-1-enyl)-9-borabicyclo[3.3.1]nonane (5a)}

Following the method of Soderquist [47], propyne $(6-10 \mathrm{~mL})$ was condensed in a Schlenk flask, to which was added 9-BBN (0.5 M in THF, $3.76 \mathrm{~g}, 60.0 \mathrm{~mL}, 30.0 \mathrm{mmol})$. After addition the mixture was stirred at $0{ }^{\circ} \mathrm{C}$ until the white solid disappeared. Two hours later, the excess propyne and solvent were removed under reduced pressure, and the residue was subjected to high vacuum ( $0.1 \mathrm{mbar})$ through a dry ice-acetone condenser. The low boiling point component (the literature reported boiling point of 
was $66^{\circ} \mathrm{C} / 0.9$ Torr) [47] was collected as a colourless oil (2.95 g, 56\%). All spectral data matched those reported in the literature [45]. ${ }^{1} \mathrm{H}-\mathrm{NMR}\left(300 \mathrm{MHz}, \mathrm{CDCl}_{3}\right) \delta: 6.80(1 \mathrm{H}, \mathrm{dq}, J=17.2,6.4 \mathrm{~Hz}$, $\left.\mathrm{HC}=\mathrm{CHCH}_{3}\right), 6.23(1 \mathrm{H}, \mathrm{dq}, J=17.2,1.5 \mathrm{~Hz}, \mathrm{BCH}=), 1.94\left(3 \mathrm{H}, \mathrm{dd}, J=6.4,1.5 \mathrm{~Hz}, \mathrm{CH}_{3}\right), 1.90-1.79$ $\left(6 \mathrm{H}\right.$, br. m, $\left.3 \times \mathrm{CH}_{2}\right), 1.72-1.60\left(6 \mathrm{H}, \mathrm{m}, 3 \times \mathrm{CH}_{2}\right), 1.28-1.17(2 \mathrm{H}, \mathrm{m}, 2 \times \mathrm{CH}) ;{ }^{13} \mathrm{C}-\mathrm{NMR}(75 \mathrm{MHz}$, $\left.\mathrm{CDCl}_{3}\right) ; \delta: 151.0(2 \times \mathrm{CH}=\mathrm{CH}), 33.8(2 \times \mathrm{CH}), 24.6\left(2 \times \mathrm{CH}_{2}\right), 23.6\left(4 \times \mathrm{CH}_{2}\right), 22.1\left(\mathrm{CH}_{3}\right) ; \mathrm{HRMS}$ (CI) calcd. for $\mathrm{C}_{11} \mathrm{H}_{20}{ }^{11} \mathrm{~B}$ : 163.1580; Found: 163.1582; ${ }^{11} \mathrm{~B}-\mathrm{NMR}(96 \mathrm{MHz}, \mathrm{CDCl} 3) 77$.

\subsection{B-(trans-1-Hexenyl)-9-borabicyclo(3,3,1)nonane (5b)}

Following the method of Brown [48], 9-BBN (0.5 M in THF, $40.0 \mathrm{~mL}, 20.0 \mathrm{mmol}$ ) was added drop-wise to pre-degassed 1-hexyne $(3.60 \mathrm{~g}, 5.0 \mathrm{~mL}, 44.0 \mathrm{mmol})$ at $0{ }^{\circ} \mathrm{C}$. After addition, the reaction mixture was warmed to room temperature and stirred for two hours. The solvent was removed under reduced pressure and the crude product was purified by distillation under reduced pressure to give the borane as a pale yellow oil (2.58 g, 60\%). B.p. 83-90 ${ }^{\circ} \mathrm{C} / 0.15 \mathrm{mbar}$ (lit [48] B.p. $72-74{ }^{\circ} \mathrm{C} / 0.03$ mbar). All spectral data matched those reported in the literature [45,48]. ${ }^{1} \mathrm{H}-\mathrm{NMR}\left(400 \mathrm{MHz}, \mathrm{CDCl}_{3}\right) ; \delta: 6.83$ $\left(1 \mathrm{H}, \mathrm{dt}, J=17.2,6.4 \mathrm{~Hz}, \mathrm{HC}=\mathrm{CHCH}_{2}\right), 6.24(1 \mathrm{H}, \mathrm{dt}, J=17.2,1.5 \mathrm{~Hz}, \mathrm{BCH}=\mathrm{CH}), 2.28(2 \mathrm{H}, \mathrm{dt}, J=7.0$, $\left.6.4 \mathrm{~Hz}, \mathrm{CH}_{2} \mathrm{CH}=\mathrm{CH}\right), 1.91-1.84\left(6 \mathrm{H}, \mathrm{m}, 3 \times \mathrm{CH}_{2}\right), 1.77-1.67\left(6 \mathrm{H}, \mathrm{m}, 3 \times \mathrm{CH}_{2}\right), 1.51-1.43(2 \mathrm{H}, \mathrm{m}$, $2 \times \mathrm{CH}), 1.41-1.33\left(2 \mathrm{H}, \mathrm{m}, \mathrm{CH}_{2}\right), 1.31-1.22\left(2 \mathrm{H}, \mathrm{m}, \mathrm{CH}_{2} \mathrm{CH}_{3}\right), 0.92\left(3 \mathrm{H}, \mathrm{t}, J=7.2 \mathrm{~Hz}, \mathrm{CH}_{3}\right)$; ${ }^{13} \mathrm{C}-\mathrm{NMR}\left(75 \mathrm{MHz}, \mathrm{CDCl}_{3}\right) ; \delta: 156.3(2 \times \mathrm{CH}=\mathrm{CH}), 35.9(2 \times \mathrm{CH}), 33.8\left(2 \times \mathrm{CH}_{2}\right), 33.7\left(4 \times \mathrm{CH}_{2}\right)$ $30.7\left(\mathrm{CH}_{2}\right), 23.6\left(\mathrm{CH}_{2}\right), 22.5\left(\mathrm{CH}_{2}\right), 14.1\left(\mathrm{CH}_{3}\right)$; HRMS $(\mathrm{CI})$ calcd. for $\mathrm{C}_{14} \mathrm{H}_{26}{ }^{11} \mathrm{~B}$ : 205.2049; Found: 205.2050; ${ }^{11} \mathrm{~B}-\mathrm{NMR}\left(96 \mathrm{MHz}, \mathrm{CDCl}_{3}\right) 77$.

\section{5. (R)-3-((S)-2,2-Dimethyl-1,3-dioxolan-4-yl)-1-hydroxy-1-phenylpropan-2-yl}

Diisopropylcarbamate (4a-I)

To a solution of carbamate $(0.208 \mathrm{mg}, 0.75 \mathrm{mmol})$ in $\mathrm{Et}_{2} \mathrm{O}(5 \mathrm{~mL})$ at $-78{ }^{\circ} \mathrm{C}$ was added $s \mathrm{BuLi}(1.3 \mathrm{M}$ in cyclohexane, $0.84 \mathrm{~mL}, 1.05 \mathrm{mmol}$,) drop-wise. This mixture was then stirred at $-78{ }^{\circ} \mathrm{C}$ for $5 \mathrm{~h}$ followed by addition of benzaldehyde $(2.00 \mathrm{~mL}, 2.00 \mathrm{mmol})$. The reaction was stirred for $2 \mathrm{~h}$ and then warmed to r.t. and stirred for $12 \mathrm{~h}$. The reaction mixture was then cooled to $0{ }^{\circ} \mathrm{C}$ and a solution of $2 \mathrm{~N}$ $\mathrm{HCl}(10 \mathrm{~mL})$ was added drop-wise. The layers were separated and the aqueous layer was extracted with $\mathrm{Et}_{2} \mathrm{O}(3 \times 15 \mathrm{~mL})$. The combined organic layers were dried over $\mathrm{MgSO}_{4}$ and concentrated in vacuo. The crude product with four pairs of diastereomers was purified by flash chromatography $\left(\mathrm{SiO}_{2}, 10 \%\right.$ EtOAc/petroleum ether) to give the major diastrereomeric product 4a-I $(196 \mathrm{mg}, 84 \%$, d.r. $=98: 2)$ as a colourless oil. Here the stereochemical identity for a carbon bearing the hydroxyl group shown with squiggly line is unspecified. This might be due to the possible attack of the lithiated intermediate on faces of benzadehyde. Secondly, in the ${ }^{1} \mathrm{H}-\mathrm{NMR}$ spectrum, the proton coupling at this position is not clear hence it is given as a multiplet. Spectral data matched those reported in the literature [3]. $R_{\mathrm{f}}=0.40$ $\left(10 \%\right.$ EtOAc in petroleum ether); IR $v_{\max }$ (neat) $/ \mathrm{cm}^{-1} 3447,2970,1680,1442,1314,1070 ;{ }^{1} \mathrm{H}-\mathrm{NMR}$ $\left(\mathrm{CDCl}_{3}, 270 \mathrm{MHz}\right) ; \delta$ : 7.30-7.19 (5 H, m, arom. H), 5.93-5.88 (1 H, m, CH(OH)Ph), 4.42-4.30 (1 H, m, CHOCb), $4.21\left(2 \mathrm{H}, \mathrm{d}, J=5.7 \mathrm{~Hz}, \mathrm{CH}_{2} \mathrm{OC}\right), 3.98-3.61(2 \mathrm{H}, \mathrm{m}, 2 \times \mathrm{CH}), 3.58-3.51(1 \mathrm{H}, \mathrm{m}, \mathrm{CH})$, $3.47(1 \mathrm{H}$, br s, $\mathrm{OH}), 1.92\left(2 \mathrm{H}, \mathrm{dd}, J=8.9,4.8 \mathrm{~Hz}, \mathrm{CH}\right.$ ), 1.37 (3 H, s, $\left.\mathrm{CH}_{3}\right), 1.31\left(3 \mathrm{H}, \mathrm{s}, \mathrm{CH}_{3}\right), 1.16$ $\left(12 \mathrm{H}, \mathrm{d}, J=6.9 \mathrm{~Hz}, 4 \times \mathrm{CH}_{3}\right) ;{ }^{13} \mathrm{C}-\mathrm{NMR}\left(\mathrm{CDCl}_{3}, 100 \mathrm{MHz}\right) ; \delta: 155.5(C=\mathrm{O}), 133.2(C-\mathrm{Ar}), 130.1$ $(2 \times \mathrm{CH}-\mathrm{Ar}), 129.8(2 \times \mathrm{CH}-\mathrm{Ar}), 128.5(\mathrm{CH}-\mathrm{Ar}), 119.2\left(\mathrm{C}_{\left(\mathrm{CH}_{3}\right)}\right), 73.6(\mathrm{CHOH}), 66.6(\mathrm{CHOCb}), 64.6$ 
$\left(\mathrm{CHCH}_{2} \mathrm{O}\right), 60.9\left(\mathrm{CH}_{2} \mathrm{O}\right), 45.6(2 \times \mathrm{CHN}), 33.3\left(\mathrm{CH}_{2}\right), 25.7\left(\mathrm{CH}_{3}\right), 21.3\left(\mathrm{CH}_{3}\right), 20.6\left(4 \times \mathrm{CH}_{3}\right)$; HRMS (ESI) calcd. for $\mathrm{C}_{21} \mathrm{H}_{33} \mathrm{NO}_{5} \mathrm{Na}(\mathrm{M}+\mathrm{Na})$ : 389.2359; Found: 389.2364; $[\alpha]_{D}^{23}-17.2$ (conc. 1.1, $\mathrm{CH}_{3} \mathrm{OH}$ ); Anal. Calcd for $\mathrm{C}_{21} \mathrm{H}_{33} \mathrm{NO}_{5}$ : C, 66.46; H, 8.76; N, 3.69; Found: C, 66.50; H, 8.72; N, 3.70.

\section{6. (R)-3-((S)-2,2-Dimethyl-1,3-dioxolan-4-yl)-1-hydroxy-1,1-diphenylpropan-2-yldiisopropyl- carbamate (4a-III)}

To a solution of carbamate $(0.208 \mathrm{mg}, 0.75 \mathrm{mmol})$ in $\mathrm{Et}_{2} \mathrm{O}(5 \mathrm{~mL})$ at $-78^{\circ} \mathrm{C}$ was added $s \mathrm{BuLi}(1.3 \mathrm{M}$ in cyclohexane, $0.84 \mathrm{~mL}, 1.05 \mathrm{mmol}$,) drop-wise. This mixture was then stirred at $-78{ }^{\circ} \mathrm{C}$ for $5 \mathrm{~h}$ followed by addition of benzophenone $(1.75 \mathrm{~mL}, 2.00 \mathrm{mmol})$. The reaction was stirred for $4 \mathrm{~h}$ and then warmed to r.t. and stirred for $12 \mathrm{~h}$. The reaction mixture was then cooled to $0{ }^{\circ} \mathrm{C}$ and a solution of $2 \mathrm{~N}$ $\mathrm{HCl}(10 \mathrm{~mL})$ was added drop-wise. The layers were separated and the aqueous layer was extracted with $\mathrm{Et}_{2} \mathrm{O}(3 \times 15 \mathrm{~mL})$. The combined organic layers were dried over $\mathrm{MgSO}_{4}$ and concentrated in vacuo. The crude product with 4 diastereomers was purified by flash chromatography $\left(\mathrm{SiO}_{2}, 10 \%\right.$ EtOAc/petroleum ether) to give the major diastereomeric product 4a-III $(170 \mathrm{mg}, 84 \%$, d.r. $=98: 2)$ as a colourless oil. Spectral data matched those reported in the literature [3]. $R_{\mathrm{f}}=0.35(10 \%$ EtOAc in petroleum ether); IR $v_{\max }$ (neat) $/ \mathrm{cm}^{-1} 3432,2890,1690,1438,1060 ;{ }^{1} \mathrm{H}-\mathrm{NMR}\left(\mathrm{CDCl}_{3}, 270 \mathrm{MHz}\right)$; $\delta$ : 7.28-7.09 (10 H, m, arom. H), 4.35-4.31 (1 H, m, CHOCb), $4.11\left(2 \mathrm{H}, \mathrm{d}, J=5.4 \mathrm{~Hz}, \mathrm{CH}_{2} \mathrm{OC}\right)$, 3.96-3.70 (2 H, m, CH), 3.52-3.46 (1 H, m, CH), $2.90(1 \mathrm{H}$, br s, OH), $1.91(2 \mathrm{H}, \mathrm{dd}, J=8.6,4.9 \mathrm{~Hz}$, $\left.\mathrm{CH}_{2}\right), 1.35(3 \mathrm{H}, \mathrm{s}, \mathrm{CH}), 1.29(3 \mathrm{H}, \mathrm{s}, \mathrm{CH}), 1.14\left(12 \mathrm{H}, \mathrm{d}, \mathrm{J}=6.9 \mathrm{~Hz}, 4 \times \mathrm{CH}_{3}\right) ;{ }^{13} \mathrm{C}-\mathrm{NMR}\left(\mathrm{CDCl}_{3}\right.$, $100 \mathrm{MHz}) ; \delta: 153.0(\mathrm{C}=\mathrm{O}), 145.2(2 \times \mathrm{C}-\mathrm{Ar}), 129.8(4 \times \mathrm{CH}-\mathrm{Ar}), 128.5(4 \times \mathrm{CH}-\mathrm{Ar}), 119.2\left(\mathrm{C}\left(\mathrm{CH}_{3}\right)_{2}\right)$, $83.6(\mathrm{C}-\mathrm{OH}), 82.0(\mathrm{CHOCb}), 71.6\left(\mathrm{CHCH}_{2} \mathrm{O}\right), 60.9\left(\mathrm{CH}_{2} \mathrm{O}\right), 45.6(2 \times \mathrm{CHN}), 33.3\left(\mathrm{CH}_{2}\right), 25.7\left(\mathrm{CH}_{3}\right)$, $21.3\left(\mathrm{CH}_{3}\right), 20.6\left(4 \times \mathrm{CH}_{3}\right)$; HRMS (ESI) calcd. for $\mathrm{C}_{27} \mathrm{H}_{37} \mathrm{NO}_{5} \mathrm{Na}(\mathrm{M}+\mathrm{Na})$ : 458.2031; Found: 458.2060; $[\alpha]_{D}^{23}+27.3$ (conc. $\left.1.5, \mathrm{CH}_{3} \mathrm{OH}\right)$.

\section{7. (S)-2-((S)-2,2-Dimethyl-1,3-dioxolan-4-yl)-1-(trimethylstannyl)ethyl Diisopropylcarbamate (4a-II)}

To a solution of carbamate $(0.208 \mathrm{mg}, 0.75 \mathrm{mmol})$ in $\mathrm{Et}_{2} \mathrm{O}(5 \mathrm{~mL})$ at $-78{ }^{\circ} \mathrm{C}$ was added $s \mathrm{BuLi}(1.3 \mathrm{M}$ in cyclohexane/hexane, $16.2 \mathrm{~mL}, 22.0 \mathrm{mmol}$ ) drop-wise at $-78{ }^{\circ} \mathrm{C}$ followed by trapping with trimethyltin chloride ( $1 \mathrm{M}$ in hexane, $0.58 \mathrm{~mL}, 1.7 \mathrm{mmol}$ ). The crude material with 4 diastereomers was purified by flash column chromatography $\left(\mathrm{SiO}_{2}\right.$, EtOAc/petroleum ether, 1:4) to give the major diastereomer 4a-II as a colourless oil $(122 \mathrm{mg}, 78 \%$, d.r. $=98: 2)$. Spectral data matched those reported in the literature [3]. $R_{\mathrm{f}}=0.43$ (10\% EtOAc in petrol); ${ }^{1} \mathrm{H}-\mathrm{NMR}\left(\mathrm{CDCl}_{3}, 270 \mathrm{MHz}\right) ; \delta: 4.38(1 \mathrm{H}$, ddd, $J=11.3,9.2,5.2 \mathrm{~Hz}$, $\left.\mathrm{CHS}_{n}\right), 4.10\left(2 \mathrm{H}, \mathrm{d}, J=5.5 \mathrm{~Hz}, \mathrm{CH}_{2} \mathrm{OC}\left(\mathrm{CH}_{3}\right)_{2}\right), 3.70-3.60\left(1 \mathrm{H}, \mathrm{m}, \mathrm{CHCH}_{2} \mathrm{O}\right), 3.51-3.46(2 \mathrm{H}, \mathrm{m}$, $2 \times \mathrm{CH}), 1.90\left(2 \mathrm{H}, \mathrm{dd}, J=8.2,5.0 \mathrm{~Hz}, \mathrm{CH}_{2}\right), 1.28(3 \mathrm{H}, \mathrm{s}, \mathrm{CH}), 1.12\left(3 \mathrm{H}, \mathrm{s}, \mathrm{CH}_{3}\right), 1.15(12 \mathrm{H}, \mathrm{d}$, $\left.J=6.9 \mathrm{~Hz}, 4 \times \mathrm{CH}_{3}\right), 0.69\left(9 \mathrm{H}, \mathrm{s}, \mathrm{Sn}\left(\mathrm{CH}_{3}\right)_{3}\right) ;{ }^{13} \mathrm{C}-\mathrm{NMR}\left(\mathrm{CDCl}_{3}, 67.5 \mathrm{MHz}\right) ; \delta: 153.2(\mathrm{C}=\mathrm{O}), 119.2$ $\left(\mathrm{C}\left(\mathrm{CH}_{3}\right)_{2}\right), 77.6\left(\mathrm{CHCH}_{2} \mathrm{O}\right), 68.9\left(\mathrm{CH}_{2} \mathrm{O}\right), 63.0\left(\mathrm{CHS}_{n}\right), 55.5(2 \times \mathrm{CHN}), 32.4\left(\mathrm{CH}_{2}\right), 25.8\left(2 \times \mathrm{CH}_{3}\right)$, $21.4\left(3 \times \mathrm{CH}_{3}\right), 21.2\left(4 \times \mathrm{CH}_{3}\right)$; HRMS (ESI) calcd. for $\mathrm{C}_{17} \mathrm{H}_{35} \mathrm{NO}_{4} \mathrm{SnNa}(\mathrm{M}+\mathrm{Na})$ : 437.1588; Found: 437.1590; $[\alpha]_{D}^{23}+11.3$ (conc. 1.1, $\mathrm{CH}_{3} \mathrm{OH}$ ); Anal. Calcd for $\mathrm{C}_{17} \mathrm{H}_{35} \mathrm{NO}_{4} \mathrm{Sn}$ : C, 46.81; H, 8.09; N, 3.21; Found: C, 46.80; H, 8.10; N, 3.17. 
3.8. (1R,2R,Z)-5-((R)-2,2-Dimethyl-1,3-dioxalan-4-yl)-2-methyl-1-phenylpent-3-en-1-ol (8-I)

$s \operatorname{BuLi}(1.3 \mathrm{M}$ in cyclohexane, $0.84 \mathrm{~mL}, 1.05 \mathrm{mmol})$ was added at $-78^{\circ} \mathrm{C}$ to a solution of acetonide carbamate $(208 \mathrm{mg}, 0.75 \mathrm{mmol})$. After stirring for $5 \mathrm{~h}, B$-vinyl,Me-9-BBN (1 M in Et $2 \mathrm{O}, 2.0 \mathrm{~mL}$, $1.12 \mathrm{mmol})$ was added at $-78{ }^{\circ} \mathrm{C}$, followed by trapping with benzaldehyde $(2.0 \mathrm{~mL}, 2.0 \mathrm{mmol})$ at $-78{ }^{\circ} \mathrm{C}$. The crude product was purified by flash column chromatography $\left(\mathrm{SiO}_{2}\right.$, petroleum ether/EtOAc 10:2) followed by removal of residual benzaldehyde at reduced pressure, to give the alcohol (40 mg, 56\%, d.r. $=90: 10)$ as a colourless oil. $R_{\mathrm{f}}=0.34\left(20 \%\right.$ EtOAc in petrol); $[\alpha]_{D}^{24}+10.4$ (conc. $\left.0.50, \mathrm{CH}_{2} \mathrm{Cl}_{2}\right)$; ${ }^{1} \mathrm{H}-\mathrm{NMR}\left(400 \mathrm{MHz} ; \mathrm{CDCl}_{3}\right) ; \delta: 7.37-7.28(5 \mathrm{H}, \mathrm{m}$, arom.H), $5.64(1 \mathrm{H}$, ddd, $J=10.9,6.1,6.1 \mathrm{~Hz}$, (acetonide) $\mathrm{CH}_{2} \mathrm{CH}=\mathrm{CH}$ ), $5.52\left(1 \mathrm{H}\right.$, dddd, $J=10.9,7.5,1.4,1.4 \mathrm{~Hz}$, (acetonide) $\left.\mathrm{CH}_{2} \mathrm{CH}=\mathrm{CH}\right), 4.29(1 \mathrm{H}$, $\mathrm{dd}, J=8.5,2.9 \mathrm{~Hz}, \mathrm{CHOH}), 4.17-4.12\left(1 \mathrm{H}, \mathrm{m}, \mathrm{CHCHHOC}\left(\mathrm{CH}_{3}\right)_{2}\right), 4.09(1 \mathrm{H}$, ddd, $J=7.0,2.3,0.49 \mathrm{~Hz}$, $\left.\mathrm{CHCH} H \mathrm{OC}\left(\mathrm{CH}_{3}\right)_{2}\right), 3.58\left(1 \mathrm{H}, \mathrm{dddd}, J=7.0,7.0,6.2,6.2 \mathrm{~Hz}, \mathrm{CHCH}_{2} \mathrm{OC}\left(\mathrm{CH}_{3}\right)_{2}\right), 2.90(1 \mathrm{H}, \mathrm{d}, J=2.9 \mathrm{~Hz}$, $\mathrm{OH}), 2.79\left(1 \mathrm{H}\right.$, ddq, $\left.J=8.5,7.5,6.7 \mathrm{~Hz}, \mathrm{CHCH}_{3}\right), 2.48(1 \mathrm{H}$, dddd, $J=7.0,6.1,4.9,1.4 \mathrm{~Hz}$, $\mathrm{CHHCH}=\mathrm{CH}), 2.30(1 \mathrm{H}$, dddd, $J=7.1,6.1,4.9,1.4 \mathrm{~Hz}, \mathrm{CH} H \mathrm{CH}=\mathrm{CH}), 1.50(3 \mathrm{H}, \mathrm{d}, J=0.49 \mathrm{~Hz}$, $\left.\mathrm{C}\left(\mathrm{CH}_{3} \mathrm{CH}_{3}\right)\right), 1.38\left(3 \mathrm{H}, \mathrm{d}, J=0.50 \mathrm{~Hz}, \mathrm{C}\left(\mathrm{CH}_{3} \mathrm{CH}_{3}\right)\right), 0.79\left(3 \mathrm{H}, \mathrm{t}, J=6.7 \mathrm{~Hz}, \mathrm{CH}_{3}\right) ;{ }^{13} \mathrm{C}-\mathrm{NMR}(100.5 \mathrm{MHz}$; $\left.\mathrm{CDCl}_{3}\right)$; $\delta: 143.0(C$-Ar), $135.5(C=\mathrm{C}), 128.4(2 \times C \mathrm{H}-\mathrm{Ar}), 128.3(\mathrm{CH}-\mathrm{Ar}), 127.7(2 \times C \mathrm{H}-\mathrm{Ar}), 127.1$ $(\mathrm{C}=\mathrm{C}), 126.9\left(\mathrm{C}-\left(\mathrm{CH}_{3}\right)_{2}\right), 78.9(\mathrm{CHOH}), 75.6\left(\mathrm{CH}(\mathrm{O}) \mathrm{C}\left(\mathrm{CH}_{3}\right)_{2}\right), 69.3\left(\mathrm{CH}_{2}\right), 40.6(\mathrm{CH}), 32.0\left(\mathrm{CH}_{2}\right)$, $27.0\left(\mathrm{CH}_{3}\right), 25.7\left(\mathrm{CH}_{3}\right), 17.7\left(\mathrm{CH}_{3}\right)$; IRv $v_{\max }\left(\right.$ neat $\left./ \mathrm{cm}^{-1}\right): 3421,2929,1691,1457,1036,700 ; \mathrm{MS}(\mathrm{ESI})$ m/z (\%) 299 (29.1, M+Na), 259 (12.7, M-OH), 183 (4.4), 168 (5.2); HRMS (ESI) calcd. for $\mathrm{C}_{17} \mathrm{H}_{24} \mathrm{O}_{3} \mathrm{Na}(\mathrm{M}+\mathrm{Na})$ : 299.1621; Found: 299.1617; EA Anal. Calcd. for $\mathrm{C}_{17} \mathrm{H}_{24} \mathrm{O}_{3}$ : C, 73.88; H, 8.75; Found: C, 73.85; H, 8.74. ${ }^{1} \mathrm{H}-\mathrm{NMR}$ signals for the minor product $\left(400 \mathrm{MHz} ; \mathrm{CDCl}_{3}\right)$; $\delta$ : 7.37-7.28 (5 H, $\mathrm{m}$, arom.H), 5.65-5.64 (1 H, m, (acetonide) $\left.\mathrm{CH}_{2} \mathrm{CH}=\mathrm{CH}\right), 5.61-5.60\left(1 \mathrm{H}, \mathrm{m}\right.$, (acetonide) $\left.\mathrm{CH}_{2} \mathrm{CH}=\mathrm{CH}\right)$, $4.31(1 \mathrm{H}, \mathrm{dd}, J=1.9,8.0 \mathrm{~Hz}, \mathrm{CHOH}), 4.18-4.12\left(1 \mathrm{H}, \mathrm{m}, \mathrm{CHCHHOC}\left(\mathrm{CH}_{3}\right)_{2}\right), 4.09-4.07(1 \mathrm{H}, \mathrm{m}$, $\left.\mathrm{CHCH} H \mathrm{OC}\left(\mathrm{CH}_{3}\right)_{2}\right), 3.61-3.57\left(1 \mathrm{H}, \mathrm{m}, \mathrm{CHCH}_{2} \mathrm{OC}\left(\mathrm{CH}_{3}\right)_{2}\right), 2.90(1 \mathrm{H}, \mathrm{d}, J=1.9 \mathrm{~Hz}, \mathrm{OH}), 2.79-2.61$ $\left(1 \mathrm{H}, \mathrm{m}, \mathrm{CHCH}_{3}\right), 2.53-2.51(1 \mathrm{H}, \mathrm{m}, \mathrm{CHHCH}=\mathrm{CH}), 2.43-2.41(1 \mathrm{H}, \mathrm{m}, \mathrm{CH} H \mathrm{CH}=\mathrm{CH}), 1.50-148(3 \mathrm{H}$, $\left.\mathrm{C}\left(\mathrm{CH}_{3} \mathrm{CH}_{3}\right)\right), 1.39-137\left(3 \mathrm{H}, \mathrm{m}, \mathrm{C}\left(\mathrm{CH}_{3} \mathrm{CH}_{3}\right)\right), 0.65\left(3 \mathrm{H}, \mathrm{d}, J=6.2 \mathrm{~Hz}, \mathrm{CH}_{3}\right)$

3.9. (1R,2R,Z)-1-Cyclohexyl-5-((R)-2,2-dimethyl-1,3-dioxalan-4-yl)-2-methylpent-3-en-1-ol (8-II).

$s \mathrm{BuLi}(1.3 \mathrm{M}$ in cyclohexane, $0.84 \mathrm{~mL}, 1.05 \mathrm{mmol})$ was added at $-78{ }^{\circ} \mathrm{C}$ to a solution of acetonide carbamate $(208 \mathrm{mg}, 0.75 \mathrm{mmol}$ ). After stirring for $5 \mathrm{~h}, B$-vinyl,Me-9-BBN ( $1 \mathrm{M}$ in Et $2 \mathrm{O}, 2.0 \mathrm{~mL}$, $1.12 \mathrm{mmol})$ was added at $-78{ }^{\circ} \mathrm{C}$, followed by trapping with cyclohexanecarboxaldehyde $(2.0 \mathrm{~mL}$, $2.0 \mathrm{mmol})$ at $-78{ }^{\circ} \mathrm{C}$. The crude product was purified by flash column chromatography $\left(\mathrm{SiO}_{2}\right.$, petroleum ether/EtOAc 10:2) followed by removal of residual aldehyde at reduced pressure, to give the alcohol $(48 \mathrm{mg}, 54 \%$, d.r. $=95: 5)$ as a colourless oil. $R_{\mathrm{f}}=0.38(20 \%$ EtOAc in petrol $) ;[\alpha]_{D}^{24}-19.5$ (conc. 0.49, $\mathrm{CH}_{2} \mathrm{Cl}_{2}$ ); ${ }^{1} \mathrm{H}-\mathrm{NMR}\left(400 \mathrm{MHz} ; \mathrm{C}_{6} \mathrm{D}_{6}\right) ; \delta$ : 5.44-5.35 (2H, m, (acetonide) $\mathrm{CH}_{2} \mathrm{CH}=\mathrm{CHCH}\left(\mathrm{CH}_{3}\right)$, $3.85\left(1 \mathrm{H}\right.$, dddd, $\left.J=7.7,7.7,5.9,4.7 \mathrm{~Hz}, \mathrm{CHCHHOC}\left(\mathrm{CH}_{3}\right)_{2}\right), 3.70(1 \mathrm{H}, \mathrm{dd}, J=7.7,5.9 \mathrm{~Hz}$, $\left.\mathrm{CHCH} H \mathrm{OC}\left(\mathrm{CH}_{3}\right)_{2}\right), 3.34(1 \mathrm{H}, \mathrm{dd}, J=7.7,7.7 \mathrm{~Hz}, \mathrm{CHOH}), 3.01(1 \mathrm{H}, \mathrm{dddd}, J=7.6,7.6,3.7,3.7 \mathrm{~Hz}$, $\left.\mathrm{CHCH}_{2} \mathrm{OC}\left(\mathrm{CH}_{3}\right)_{2}\right), 2.65-2.55\left(1 \mathrm{H}, \mathrm{m}, \mathrm{CHCH}_{3}\right), 2.33-2.21$ (1 H, m, CH of cyclohexane), 2.00-1.93 (2 H, $\left.\mathrm{m}, \mathrm{CH}_{2} \mathrm{CH}=\mathrm{CH}\right), 1.79-1.51\left(6 \mathrm{H}, \mathrm{m}, 3 \times \mathrm{CH}_{2}\right), 1.47\left(3 \mathrm{H}\right.$, br s, $\left.\mathrm{C}\left(\mathrm{CH}_{3} \mathrm{CH}_{3}\right)\right), 1.42-1.36\left(2 \mathrm{H}, \mathrm{m}, \mathrm{CH}_{2}\right)$, $1.30\left(3 \mathrm{H}, \mathrm{d}, J=0.48 \mathrm{~Hz}, \mathrm{C}\left(\mathrm{CH}_{3} \mathrm{CH}_{3}\right)\right), 1.28-1.16\left(4 \mathrm{H}, \mathrm{m}, 2 \times \mathrm{CH}_{2}\right), 0.86\left(3 \mathrm{H}, \mathrm{d}, J=6.7 \mathrm{~Hz}, \mathrm{CH}_{3}\right)$; ${ }^{13} \mathrm{C}-\mathrm{NMR}\left(100.5 \mathrm{MHz} ; \mathrm{C}_{6} \mathrm{D}_{6}\right) ; \delta: 135.2(\mathrm{C}=\mathrm{C}), 125.8(\mathrm{C}=C), 108.9\left(C\left(\mathrm{CH}_{3}\right) 2\right), 78.7(\mathrm{CH}), 75.4(\mathrm{CH})$, 
$69.1\left(\mathrm{CH}_{2}\right), 40.3(\mathrm{CH}), 35.1(\mathrm{CH}), 32.0\left(\mathrm{CH}_{2}\right), 30.7\left(\mathrm{CH}_{2}\right), 26.9\left(\mathrm{CH}_{2}\right), 26.8\left(2 \times \mathrm{CH}_{2}\right), 26.5\left(\mathrm{CH}_{2}\right)$, $26.2\left(\mathrm{CH}_{3}\right), 25.6\left(\mathrm{CH}_{3}\right), 17.4\left(\mathrm{CH}_{3}\right)$; IR $v_{\max }\left(\right.$ neat $\left./ \mathrm{cm}^{-1}\right): 3431,1608,1496,1452,1029,962 ; \mathrm{MS}(\mathrm{ESI})$ m/z (\%) 305 (100, M+Na), 296 (21.3), 207 (8.2), 152 (7.0), 101 (6.9); HRMS (ESI) calcd. for $\mathrm{C}_{17} \mathrm{H}_{30} \mathrm{O}_{3} \mathrm{Na}(\mathrm{M}+\mathrm{Na})$ : 305.2089; Found: 305.2087; EA Anal. Calcd. for $\mathrm{C}_{17} \mathrm{H}_{30} \mathrm{O}_{3}$ : C, 72.30; H, 10.71; Found: C, 72.63; H, 10.46; ); ${ }^{1} \mathrm{H}-\mathrm{NMR}$ signals for the minor product (400 MHz; $\left.\mathrm{C}_{6} \mathrm{D}_{6}\right)$; $\delta: 5.48-5.44$ (2H, m, (acetonide) $\mathrm{CH}_{2} \mathrm{CH}=\mathrm{CHCH}\left(\mathrm{CH}_{3}\right), 3.87-3.85\left(1 \mathrm{H}, \mathrm{m}, \mathrm{CHCHHOC}\left(\mathrm{CH}_{3}\right) 2\right), 3.70(1 \mathrm{H}, \mathrm{dd}, J=7.4$, $\left.5.9 \mathrm{~Hz}, \mathrm{CHCH} H \mathrm{OC}\left(\mathrm{CH}_{3}\right)_{2}\right), 3.34(1 \mathrm{H}, \mathrm{dd}, J=5.7,7.9 \mathrm{~Hz}, \mathrm{CHOH}), 3.03-3.02(1 \mathrm{H}, \mathrm{m}$, $\left.\mathrm{CHCH}_{2} \mathrm{OC}\left(\mathrm{CH}_{3}\right)_{2}\right), 2.69-2.66\left(1 \mathrm{H}, \mathrm{m}, \mathrm{CHCH}_{3}\right), 2.35-2.33$ (1 H, m, CH of cyclohexane), 2.02-2.00 (2 H, m, $\left.\mathrm{CH}_{2} \mathrm{CH}=\mathrm{CH}\right), 1.85-1.80\left(6 \mathrm{H}, \mathrm{m}, 3 \times \mathrm{CH}_{2}\right), 1.48\left(3 \mathrm{H}\right.$, br s, $\left.\mathrm{C}\left(\mathrm{CH}_{3} \mathrm{CH}_{3}\right)\right), 1.42-1.36\left(2 \mathrm{H}, \mathrm{m}, \mathrm{CH}_{2}\right)$, $1.31\left(3 \mathrm{H}, \mathrm{s}, \mathrm{C}\left(\mathrm{CH}_{3} \mathrm{CH}_{3}\right)\right), 1.36-1.33\left(4 \mathrm{H}, \mathrm{m}, 2 \times \mathrm{CH}_{2}\right), 0.88\left(3 \mathrm{H}, \mathrm{d}, J=6.8 \mathrm{~Hz}, \mathrm{CH}_{3}\right)$.

\subsection{0. (4R,5S,Z)-1-((R)-2,2-Dimethyl-1,3-dioxolan-4-yl)-4-methylnon-2-en-5-ol (8-III)}

$s \mathrm{BuLi}(1.3 \mathrm{M}$ in cyclohexane, $0.80 \mathrm{~mL}, 1.00 \mathrm{mmol})$ was added at $-78{ }^{\circ} \mathrm{C}$ to a solution of acetonide carbamate $(200 \mathrm{mg}, 0.70 \mathrm{mmol})$. After stirring for $5 \mathrm{~h}, B$-vinyl,Me-9-BBN ( $1 \mathrm{M}$ in Et $2 \mathrm{O}, 1.6 \mathrm{~mL}$, $1.00 \mathrm{mmol})$ was added at $-78{ }^{\circ} \mathrm{C}$, followed by trapping with $n$-butyraldehyde $(2.0 \mathrm{~mL}, 2.0 \mathrm{mmol})$ at $-78{ }^{\circ} \mathrm{C}$. The crude product was purified by flash column chromatography $\left(\mathrm{SiO}_{2}\right.$, petroleum ether/EtOAc 10:2) followed by removal of residual aldehyde at reduced pressure, to give the alcohol $(57 \mathrm{mg}, 60 \%$, d.r. $=92: 8)$ as a colourless oil. $R_{\mathrm{f}}=0.36\left(20 \%\right.$ EtOAc in petroleum ether); $[\alpha]_{D}^{24}+21.9$ (conc. 0.50, $\left.\mathrm{CH}_{2} \mathrm{Cl}_{2}\right)$; ${ }^{1} \mathrm{H}-\mathrm{NMR}\left(400 \mathrm{MHz} ; \mathrm{CDCl}_{3}\right) ; \delta: 5.54(1 \mathrm{H}, \mathrm{ddd}, J=10.5,7.1,7.1 \mathrm{~Hz}$, (acetonide) $\left.\mathrm{CH}_{2} \mathrm{CH}=\mathrm{CH}\right), 5.52\left(1 \mathrm{H}\right.$, dddd, $J=10.4,7.4,1.3,1.3 \mathrm{~Hz}$, (acetonide) $\left.\mathrm{CH}_{2} \mathrm{CH}=\mathrm{CH}\right), 4.27$ (1 $\mathrm{H}, \mathrm{dd}, J=8.3,2.5 \mathrm{~Hz}, \mathrm{CHOH}), 4.15-4.11\left(1 \mathrm{H}, \mathrm{m}, \mathrm{CHCHHOC}\left(\mathrm{CH}_{3}\right)_{2}\right), 4.05(1 \mathrm{H}, \mathrm{ddd}, J=6.8,2.5$, $\left.0.56 \mathrm{~Hz}, \mathrm{CHCH} H \mathrm{OC}\left(\mathrm{CH}_{3}\right)_{2}\right), 3.58\left(1 \mathrm{H}, \mathrm{dddd}, J=6.9,6.9,6.1,6.1 \mathrm{~Hz}, \mathrm{CHCH}_{2} \mathrm{OC}\left(\mathrm{CH}_{3}\right)_{2}\right), 2.90(1 \mathrm{H}$, $\mathrm{d}, J=2.8 \mathrm{~Hz}, \mathrm{OH}), 2.79-2.74\left(1 \mathrm{H}, \mathrm{m}, \mathrm{CHCH}_{3}\right), 2.48(1 \mathrm{H}$, dddd, $J=7.0,6.1,4.9,1.4 \mathrm{~Hz}$, $\mathrm{CHHCH}=\mathrm{CH}), 2.30(1 \mathrm{H}$, dddd, $J=7.1,6.1,4.9,1.4 \mathrm{~Hz}, \mathrm{CH} H \mathrm{CH}=\mathrm{CH}), 1.59(3 \mathrm{H}, \mathrm{d}, J=0.45 \mathrm{~Hz}$, $\left.\mathrm{C}\left(\mathrm{CH}_{3} \mathrm{CH}_{3}\right)\right), 1.57-1.50\left(6 \mathrm{H}, \mathrm{m}, \mathrm{CH}_{3}\left(\mathrm{CH}_{2}\right)_{3}\right), 1.42\left(6 \mathrm{H}, \mathrm{s}, \mathrm{C}\left(\mathrm{CH}_{3} \mathrm{CH}_{3}\right)\right), 0.70\left(6 \mathrm{H}, \mathrm{m}, \mathrm{CHCH}_{3}\right.$ and $\left.\mathrm{CH}_{3}\left(\mathrm{CH}_{2}\right)_{2} \mathrm{CHOH}\right) ;{ }^{13} \mathrm{C}-\mathrm{NMR}\left(100.5 \mathrm{MHz} ; \mathrm{CDCl}_{3}\right) ; \delta: 135.0(\mathrm{C}=\mathrm{C}), 127.1(\mathrm{C}=\mathrm{C}), 126.9\left(\mathrm{C}-\left(\mathrm{CH}_{3}\right)_{2}\right)$, $78.9(\mathrm{CHOH}), 75.6\left(\mathrm{CH}(\mathrm{O}) \mathrm{C}\left(\mathrm{CH}_{3}\right)_{2}\right), 69.3\left(\mathrm{CH}_{2}\right), 40.6(\mathrm{CH}), 32.0\left(3 \times \mathrm{CH}_{2}\right), 27.0\left(\mathrm{CH}_{3}\right), 25.7\left(\mathrm{CH}_{3}\right)$, $17.7\left(2 \times \mathrm{CH}_{3}\right) ; \mathrm{IR} v_{\max }\left(\right.$ neat $\left./ \mathrm{cm}^{-1}\right): 3422,2925,1700,1460,1039,709$; MS(ESI) $\mathrm{m} / z$ (\%) 299 (29.1, $\mathrm{M}+\mathrm{Na}$ ), 259 (12.7, M-OH), 183 (4.4), 168 (5.2); HRMS (ESI) calcd. for $\mathrm{C}_{15} \mathrm{H}_{28} \mathrm{O}{ }_{3} \mathrm{Na}(\mathrm{M}+\mathrm{Na})$ : 279.2139; Found: 299.2100; EA Anal. Calcd. for $\mathrm{C}_{15} \mathrm{H}_{28} \mathrm{O}_{3}$ : C, 72.86; H, 8.73; Found: C, 72.85; H, 8.69. ${ }^{1} \mathrm{H}-\mathrm{NMR}$ signals for the minor product $\left(400 \mathrm{MHz} ; \mathrm{CDCl}_{3}\right) ; \delta: 5.56(1 \mathrm{H}$, ddd, $J=13.3,5.6,5.7 \mathrm{~Hz}$, (acetonide) $\left.\mathrm{CH}_{2} \mathrm{CH}=\mathrm{CH}\right), 5.57-5.54\left(1 \mathrm{H}, \mathrm{m}\right.$, (acetonide) $\left.\mathrm{CH}_{2} \mathrm{CH}=\mathrm{CH}\right), 4.30-4.26(1 \mathrm{H}, \mathrm{m}, \mathrm{CHOH})$, 4.16-4.15 (1 H, m, CHCHHOC $\left.\left(\mathrm{CH}_{3}\right)_{2}\right), 4.03\left(1 \mathrm{H}\right.$, ddd, $\left.J=6.3,1.9,0.80 \mathrm{~Hz}, \mathrm{CHCH} H O C\left(\mathrm{CH}_{3}\right)_{2}\right)$, $3.58\left(1 \mathrm{H}\right.$, dddd, $\left.J=7.1,7.1,5.6,5.6 \mathrm{~Hz}, \mathrm{CHCH}_{2} \mathrm{OC}\left(\mathrm{CH}_{3}\right)_{2}\right), 2.91-2.90(1 \mathrm{H}, \mathrm{m}, \mathrm{OH}), 2.83-2.80(1 \mathrm{H}$, $\left.\mathrm{m}, \mathrm{CHCH}_{3}\right), 2.48(1 \mathrm{H}, \mathrm{dddd}, J=5.8,5.6,5.4,1.8 \mathrm{~Hz}, \mathrm{CHHCH}=\mathrm{CH}), 2.32-2.31(1 \mathrm{H}, \mathrm{m}, \mathrm{CH} H \mathrm{CH}=\mathrm{CH})$,

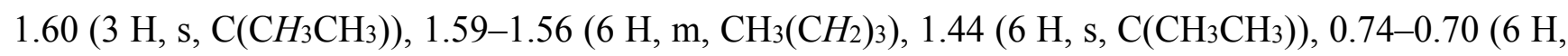
$\mathrm{m}, \mathrm{CHCH}_{3}$ and $\left.\mathrm{CH}_{3}\left(\mathrm{CH}_{2}\right)_{2} \mathrm{CHOH}\right)$. 
3.11. (1R,2R)-2-((Z)-3-((R)-2,2-Dimethyl-1,3-dioxolan-4-yl)prop-1-en-1-yl)-1phenylhexan-1-ol (8-IV).

$s \mathrm{BuLi}(1.3 \mathrm{M}$ in cyclohexane, $0.80 \mathrm{~mL}, 1.00 \mathrm{mmol})$ was added at $-78{ }^{\circ} \mathrm{C}$ to a solution of acetonide carbamate $(200 \mathrm{mg}, 0.70 \mathrm{mmol})$. After stirring for $5 \mathrm{~h}, B$-vinyl,Bu-9-BBN (1 M in Et $2 \mathrm{O}, 1.4 \mathrm{~mL}, 1.00 \mathrm{mmol}$ ) was added at $-78{ }^{\circ} \mathrm{C}$, followed by trapping with benzaldehyde $(2.0 \mathrm{~mL}, 2.0 \mathrm{mmol})$ at $-78^{\circ} \mathrm{C}$. The crude product was purified by flash column chromatography $\left(\mathrm{SiO}_{2}\right.$, petroleum ether ether/EtOAc 10:2) followed by removal of residual aldehyde at reduced pressure, to give the alcohol (44 mg, 48\%, d.r. $=90: 10)$ as a colourless oil. $R_{\mathrm{f}}=0.34\left(20 \%\right.$ EtOAc in petroleum ether); $[\alpha]_{D}^{25}-14.5$ (conc. 0.50, $\left.\mathrm{CH}_{2} \mathrm{Cl}_{2}\right){ }^{1} \mathrm{H}-\mathrm{NMR}\left(400 \mathrm{MHz} ; \mathrm{CDCl}_{3}\right) ; \delta: 7.37-7.28(5 \mathrm{H}, \mathrm{m}$, arom.H), $5.63(1 \mathrm{H}$, ddd, $J=10.9,6.0$, $6.0 \mathrm{~Hz}$, (acetonide) $\left.\mathrm{CH}_{2} \mathrm{CH}=\mathrm{CH}\right), 5.50\left(1 \mathrm{H}\right.$, dddd, $J=10.7,7.0,1.2,1.2 \mathrm{~Hz}$, (acetonide) $\left.\mathrm{CH}_{2} \mathrm{CH}=\mathrm{CH}\right)$, 4.32 (1 H, dd, $J=8.5,3.9 \mathrm{~Hz}, \mathrm{CHOH}), 4.17-4.12\left(1 \mathrm{H}, \mathrm{m}, \mathrm{CHCHHOC}\left(\mathrm{CH}_{3}\right)_{2}\right), 4.09$ (1 H, ddd, $J=7.0$, 2.3, $\left.0.49 \mathrm{~Hz}, \mathrm{CHCH} H \mathrm{OC}\left(\mathrm{CH}_{3}\right)_{2}\right), 3.58\left(1 \mathrm{H}, \mathrm{dddd}, J=7.0,7.0,6.2,6.2 \mathrm{~Hz}, \mathrm{CHCH}_{2} \mathrm{OC}\left(\mathrm{CH}_{3}\right)_{2}\right), 2.90(1 \mathrm{H}$, $\mathrm{d}, J=2.9 \mathrm{~Hz}, \mathrm{OH}), 2.62\left(1 \mathrm{H}, \mathrm{ddq}, J=8.5,7.5,6.7 \mathrm{~Hz}, \mathrm{CHCH}_{3}\right), 2.48(1 \mathrm{H}$, dddd, $J=7.0,6.1,4.9,1.4 \mathrm{~Hz}$, $\mathrm{C} H \mathrm{HCH}=\mathrm{CH}), 2.30(1 \mathrm{H}$, dddd, $J=7.1,6.1,4.9,1.4 \mathrm{~Hz}, \mathrm{CH} H \mathrm{CH}=\mathrm{CH}), 1.87-180(4 \mathrm{H}, \mathrm{m}), 1.50(2 \mathrm{H}$, t, $\left.J=0.49 \mathrm{~Hz}, \mathrm{C}\left(\mathrm{CH}_{2} \mathrm{CH}_{3}\right)\right), 1.38\left(3 \mathrm{H}, \mathrm{d}, J=0.50 \mathrm{~Hz}, \mathrm{C}\left(\mathrm{CH}_{3} \mathrm{CH}_{3}\right)\right), 0.79\left(3 \mathrm{H}, \mathrm{t}, J=6.7 \mathrm{~Hz}, \mathrm{CH}_{3}\right)$; ${ }^{13} \mathrm{C}-\mathrm{NMR}\left(100.5 \mathrm{MHz} ; \mathrm{CDCl}_{3}\right) ; \delta: 142.0(C$-Ar), $133.5(C=\mathrm{C}), 129.4(2 \times \mathrm{CH}-\mathrm{Ar}), 128.3(\mathrm{CH}-\mathrm{Ar})$, $127.7(2 \times \mathrm{CH}-\mathrm{Ar}), 127.4(\mathrm{C}=\mathrm{C}), 126.9\left(\mathrm{C}-\left(\mathrm{CH}_{3}\right)_{2}\right), 79.9(\mathrm{CHOH}), 75.6\left(\mathrm{CH}(\mathrm{O}) \mathrm{C}\left(\mathrm{CH}_{3}\right)_{2}\right), 69.3\left(4 \times \mathrm{CH}_{2}\right)$, $40.6(\mathrm{CH}), 32.0\left(4 \times \mathrm{CH}_{2}\right), 27.0\left(\mathrm{CH}_{3}\right), 25.7\left(\mathrm{CH}_{3}\right), 17.7\left(\mathrm{CH}_{3}\right) ; \mathrm{IR} v_{\max }\left(\right.$ neat $\left./ \mathrm{cm}^{-1}\right): 3400,2910,1695$, 1454, 1037, 709; HRMS (ESI) calcd. for $\mathrm{C}_{20} \mathrm{H}_{30} \mathrm{O}_{3} \mathrm{Na}(\mathrm{M}+\mathrm{Na})$ : 341.1432; Found: 341.1480; EA Anal. Calcd. for $\mathrm{C}_{20} \mathrm{H}_{30} \mathrm{O}_{3}$ : C, 71.88; H, 8.75; Found: C, 71.87; H, 8.74. ${ }^{1} \mathrm{H}-\mathrm{NMR}$ signals for the minor product (400 MHz; $\left.\mathrm{CDCl}_{3}\right) ; \delta: 7.40-7.33(5 \mathrm{H}, \mathrm{m}$, arom.H), $5.65(1 \mathrm{H}$, ddd, $J=13.9,5.2,5.2 \mathrm{~Hz}$, (acetonide) $\left.\mathrm{CH}_{2} \mathrm{CH}=\mathrm{CH}\right), 5.53\left(1 \mathrm{H}\right.$, ddd, $J=13.8,7.0,3.2 \mathrm{~Hz}$, (acetonide) $\left.\mathrm{CH}_{2} \mathrm{CH}=\mathrm{CH}\right), 4.34-4.32$ $(1 \mathrm{H}, \mathrm{m}, \mathrm{CHOH}), 4.21-4.19\left(1 \mathrm{H}, \mathrm{m}, \mathrm{CHCHHOC}\left(\mathrm{CH}_{3}\right)_{2}\right), 4.09(1 \mathrm{H}, \mathrm{ddd}, J=7.0,2.3,0.49 \mathrm{~Hz}$, $\left.\mathrm{CHCH} H \mathrm{OC}\left(\mathrm{CH}_{3}\right)_{2}\right), 3.58\left(1 \mathrm{H}, \mathrm{dddd}, J=7.1,7.1,6.1,6.0 \mathrm{~Hz}, \mathrm{CHCH}_{2} \mathrm{OC}\left(\mathrm{CH}_{3}\right)_{2}\right), 2.90(1 \mathrm{H}, \mathrm{d}, J=2.9 \mathrm{~Hz}$, $\mathrm{OH}), 2.66-2.63\left(1 \mathrm{H}, \mathrm{m}, \mathrm{CHCH}_{3}\right), 2.48(1 \mathrm{H}, \mathrm{dddd}, J=7.0,5.2,5.2,1.5 \mathrm{~Hz}, \mathrm{CHHCH}=\mathrm{CH}), 2.30(1 \mathrm{H}$, ddd, $J=7.1,5.2,5.2,1.6 \mathrm{~Hz}, \mathrm{CH} H \mathrm{CH}=\mathrm{CH}), 1.89-1.83(4 \mathrm{H}, \mathrm{m}), 1.55-1.52(2 \mathrm{H}, \mathrm{m}, J=0.51 \mathrm{~Hz}$, $\left.\mathrm{C}\left(\mathrm{CH}_{2} \mathrm{CH}_{3}\right)\right), 1.38\left(3 \mathrm{H}, \mathrm{s}, \mathrm{C}\left(\mathrm{CH}_{3} \mathrm{CH}_{3}\right)\right), 0.81\left(3 \mathrm{H}, \mathrm{t}, J=6.5 \mathrm{~Hz}, \mathrm{CH}_{3}\right)$

\subsection{2. (1S,2R)-1-Cyclohexyl-2-((Z)-3-((R)-2,2-dimethyl-1,3-dioxolan-4-yl)prop-1-en-1-yl) hexan-1-ol (8-V)}

$s \operatorname{BuLi}(1.3 \mathrm{M}$ in cyclohexane, $0.80 \mathrm{~mL}, 1.00 \mathrm{mmol})$ was added at $-78^{\circ} \mathrm{C}$ to a solution of acetonide carbamate $(200 \mathrm{mg}, 0.70 \mathrm{mmol})$. After stirring for $5 \mathrm{~h}, B$-vinyl,Bu-9-BBN ( $1 \mathrm{M}$ in Et $2 \mathrm{O}, 1.6 \mathrm{~mL}$, $1.00 \mathrm{mmol}$ ) was added at $-78{ }^{\circ} \mathrm{C}$, followed by trapping with cyclohexanecarboxaldehyde $(2.2 \mathrm{~mL}$, $2.0 \mathrm{mmol})$ at $-78{ }^{\circ} \mathrm{C}$. The crude product was purified by flash column chromatography $\left(\mathrm{SiO}_{2}\right.$, petroleum ether/EtOAc 10:2) followed by removal of residual aldehyde at reduced pressure, to give the alcohol $(42 \mathrm{mg}, 52 \%$, d.r. $=94: 6)$ as a colourless oil. $R_{\mathrm{f}}=0.38(20 \%$ EtOAc in petroleum ether); $[\alpha]_{D}^{25}-18.4$ (conc. 0.49, $\mathrm{CH}_{2} \mathrm{Cl}_{2}$ ); ${ }^{1} \mathrm{H}-\mathrm{NMR}\left(400 \mathrm{MHz} ; \mathrm{C}_{6} \mathrm{D}_{6}\right) ; \delta: 5.60-5.45(2 \mathrm{H}, \mathrm{m}$, (acetonide) $\mathrm{CH}_{2} \mathrm{CH}=\mathrm{CHCH}\left(\mathrm{CH}_{3}\right), 4.01\left(1 \mathrm{H}\right.$, dddd, $\left.J=7.7,7.7,5.9,4.7 \mathrm{~Hz}, \mathrm{CHCHHOC}\left(\mathrm{CH}_{3}\right)_{2}\right), 3.70(1 \mathrm{H}, \mathrm{dd}$, $\left.J=7.7,5.9 \mathrm{~Hz}, \mathrm{CHCH} H \mathrm{OC}\left(\mathrm{CH}_{3}\right)_{2}\right), 3.33(1 \mathrm{H}, \mathrm{dd}, J=7.7,7.7 \mathrm{~Hz}, \mathrm{CHOH}), 3.00(1 \mathrm{H}, \mathrm{dddd}, J=7.6$, 7.6, 3.7, $\left.3.7 \mathrm{~Hz}, \mathrm{CHCH}_{2} \mathrm{OC}\left(\mathrm{CH}_{3}\right)_{2}\right), 2.75-2.55$ (1 H, m, $\left.\mathrm{CHCH}_{3}\right), 2.38-2.26(1 \mathrm{H}, \mathrm{m}, \mathrm{CH}$ of 
cyclohexane), 2.10-1.98 (2 H, m, $\left.\mathrm{CH}_{2} \mathrm{CH}=\mathrm{CH}\right), 1.79-1.51\left(6 \mathrm{H}, \mathrm{m}, 3 \times \mathrm{CH}_{2}\right), 1.45(3 \mathrm{H}$, br s, $\left.\mathrm{C}\left(\mathrm{CH}_{3} \mathrm{CH}_{3}\right)\right), 1.42-1.34\left(4 \mathrm{H}, \mathrm{m}, \mathrm{CH}_{2}\right), 1.30\left(3 \mathrm{H}, \mathrm{d}, J=0.48 \mathrm{~Hz}, \mathrm{C}\left(\mathrm{CH}_{3} \mathrm{CH}_{3}\right)\right), 1.28-1.13(6 \mathrm{H}, \mathrm{m}$, $\left.2 \times \mathrm{CH}_{2}\right), 0.86\left(3 \mathrm{H}, \mathrm{d}, J=6.7 \mathrm{~Hz}, \mathrm{CH}_{3}\right) ;{ }^{13} \mathrm{C}-\mathrm{NMR}\left(100.5 \mathrm{MHz} ; \mathrm{C}_{6} \mathrm{D}_{6}\right) ; ; \delta: 136.2(\mathrm{C}=\mathrm{C}), 126.8(\mathrm{C}=\mathrm{C})$, $109.9\left(\mathrm{C}\left(\mathrm{CH}_{3}\right)_{2}\right), 79.7(\mathrm{CH}), 75.4(\mathrm{CH}), 69.1\left(\mathrm{CH}_{2}\right), 40.3(\mathrm{CH}), 35.1(\mathrm{CH}), 32.0\left(\mathrm{CH}_{2}\right), 30.7\left(\mathrm{CH}_{2}\right), 26.9$ $\left(\mathrm{CH}_{2}\right), 26.8\left(2 \times \mathrm{CH}_{2}\right), 26.5\left(\mathrm{CH}_{2}\right), 26.2\left(\mathrm{CH}_{3}\right), 25.6\left(\mathrm{CH}_{3}\right), 17.4\left(\mathrm{CH}_{3}\right) ; \mathrm{IR} v_{\max }\left(\right.$ neat $\left./ \mathrm{cm}^{-1}\right): 3441$, 1608, 1496, 1470, 1029, 962; HRMS (ESI) calcd. for $\mathrm{C}_{20} \mathrm{H}_{36} \mathrm{O}_{3} \mathrm{Na}(\mathrm{M}+\mathrm{Na})$ : 347.2065; Found: 347.2060; EA Anal. Calcd. for $\mathrm{C}_{20} \mathrm{H}_{36} \mathrm{O}_{3}$ : C, 73.31; H, 10.01; Found: C, 71.33; H, 10.06. ${ }^{1} \mathrm{H}-\mathrm{NMR}$ signals for the minor product $\left(400 \mathrm{MHz} ; \mathrm{C}_{6} \mathrm{D}_{6}\right) ; \delta$ : 5.66-5.61 (2 H, m, (acetonide) $\mathrm{CH}_{2} \mathrm{CH}=\mathrm{CHCH}\left(\mathrm{CH}_{3}\right)$, 4.07-4.04 (1 H, m, CHCHHOC(CH3)2), $3.74\left(1 \mathrm{H}, \mathrm{dd}, J=7.4,5.0 \mathrm{~Hz}, \mathrm{CHCH} H \mathrm{OC}\left(\mathrm{CH}_{3}\right)_{2}\right), 3.36-3.34$ $(1 \mathrm{H}, \mathrm{m}, \mathrm{CHOH}), 3.02\left(1 \mathrm{H}, \mathrm{dddd}, J=7.2,7.2,4.1,3.7 \mathrm{~Hz}, \mathrm{CHCH}_{2} \mathrm{OC}\left(\mathrm{CH}_{3}\right)_{2}\right), 2.75-2.55(1 \mathrm{H}, \mathrm{m}$, $\left.\mathrm{CHCH}_{3}\right), 2.46-2.39$ (8 H,m, $\mathrm{CH}$ of cyclohexane), 2.11-1.98 (2 H, m, $\left.\mathrm{CH}_{2} \mathrm{CH}=\mathrm{CH}\right), 1.47(3 \mathrm{H}$, br s, $\left.\mathrm{C}\left(\mathrm{CH}_{3} \mathrm{CH}_{3}\right)\right), 1.42-1.34\left(4 \mathrm{H}, \mathrm{m}, \mathrm{CH}_{2}\right), 1.30\left(3 \mathrm{H}, \mathrm{d}, J=0.48 \mathrm{~Hz}, \mathrm{C}\left(\mathrm{CH}_{3} \mathrm{CH}_{3}\right)\right), 1.30-1.28(2 \mathrm{H}, \mathrm{m}$, $\left.2 \times \mathrm{CH}_{2}\right), 0.86\left(3 \mathrm{H}, \mathrm{t}, J=6.2 \mathrm{~Hz}, \mathrm{CH}_{3}\right)$.

\section{Conclusions}

The lithiation of 2-(2,2-dimethyl-1,3-dioxolan-4-yl)ethyl diisopropylcarbamate (1) can be achieved with extreme selectivity by the selection of appropriate chelating agents. Furthermore, this application of chelation-directed asymmetric lithiation and subsequent borylation and allylation reactions then gave (Z)-anti-homoallylic alcohols $\mathbf{8}(\mathbf{I}-\mathbf{V})$ in good yield and excellent $d . r$. with full stereocontrol.

\section{Supplementary Materials}

Supplementary materials can be accessed at: http://www.mdpi.com/1420-3049/20/06/9890/s1.

\section{Acknowledgments}

Adeem Mahmood gratefully acknowledge the Higher Education Commission of Pakistan and are thankful to Varinder K. Aggarwal (University of Bristol, Bristol, UK). This project was supported by King Saud University, Deanship of Scientific Research, College of Science, Research Centre.

\section{Author Contributions}

Adeem Mahmood designed the research; Adeem Mahmood and Saima Parvez performed the research; Hamad Z. Alkhathlan, Merajuddin Khan and Sohail A. Shahzad analyzed the data; Adeem Mahmood wrote the paper. Hamad Z. Alkhathlan supervised the manuscript; All authors read and approved the final manuscript.

\section{Conflicts of Interest}

The authors declare no conflict of interest. 


\section{References}

1. Hoppe, D.; Hintze, F.; Tebben, P.; Paetow, M.; Ahrens, H.; Schwerdtfeger, J.; Sommerfeld, P.; Haller, J.; Guarnieri, S.; Kolczewski, S.; et al. Enantioslective synthesis via sparteine-induced asymmetric deprotonation. Pure Appl. Chem. 1994, 66, 1479-1486.

2. Hoppe, D.; Hintze, F.; Tebben, P. Chirale Lithium-1-oxyalkanide durch asymmetrische Deprotonierung; enantioselective Synthese von 2-Hydroxyalkansäuren und sekundären Alkanolen. Angew. Chem. Int. Ed. 1990, 102, 1457-1459.

3. Helmke, H.; Hoppe, D. Chelation directed asymmetric lithiation and C-substituion of 1,2,4-butanetriol acetonide. Synlett 1995, 978-980, doi:10.1055/s-1995-5115.

4. Paulsen, H.; Graeve, C.; Hoppe, D. Convenient Synthesis of Enantiomerically Enriched (Oxybutenyl)stannanes and Their Lewis Acid Catalyzed Homoaldol Reactions. Synthesis 1996, 141-144, doi:10.1055/s-1996-4172.

5. Basu, A.; Thayumanavan, S. Configurational stability and transfer of stereochemical information in the reactions of enantioenriched organolithium reagents. Angew. Chem. Int. Ed. 2002, 41, 716-738.

6. Stymiest, J.L.; Dutheuil, G.; Mahmood, A.; Aggarwal, V.K. Lithiated carbamates: Iterative homologation of boranes and boronic esters. Angew. Chem. Int. Ed. 2007, 46, 7491-7495.

7. Sommerfeld, P.; Hoppe, D. Enantioselective generation of 3-amino-1-oxy-substituted carbanions by sparteine-induced deprotonation: Asymmetric synthesis of 3-hydroxy-alkylamines. Synlett 1992, 764-766, doi:10.1055/s-1992-21487.

8. Guarnieri, W.; Grehl, M.; Hoppe, D. Regio and stereoselective electrophilic C-substituion of 2-(N,N-dibenzylamino)-1,v-alkanediols by lithiation of the their carbamates. Angew. Chem. Int. Ed. 1994, 106, 1815-1818.

9. Stymiest, J.L.; Bagutski, V.; French, R.M.; Aggarwal, V.K. Highly enantioselective conversion of secondary alcohols into tertiary alcohols with broad scope. Nature 2008, 456, 778-784.

10. Dutheuil, G.; Webster, M.P.; Worthington, P.A.; Aggarwal, V.K. Stereocontrolled synthesis of carbon chains bearing contiguous methyl groups by iterative boronic ester homologations: Application to the total synthesis of (+)-Faranal. Angew. Chem. Int. Ed. 2009, 48, 6179-6183.

11. Bonet, A.; Odachowski, M.; Leonori, D.; Essafi, S.; Varinder Aggarwal, V.K.; Enantiospecific $\mathrm{sp}^{2}-\mathrm{sp}^{3}$ coupling of secondary and tertiary boronic esters. Nat. Chem. 2014, 6, 584-589.

12. Rasappan, R.; Aggarwal, V.K. Synthesis of hydroxyphthioceranic acid using a traceless lithiationborylation-protodeboronation strategy. Nat. Chem. 2014, 6, 810-814.

13. Burns, M.; Essafi, S.; Bame, J.R.;Bull, S.P.; Webster, M.P.; Balieu, S.; Dale, J.W.; Butts, C.P.; Harvey, J.N.; Aggarwal, V.K. Assembly-line synthesis of organic molecules with tailored shapes. Nature 2014, 513, 183-188.

14. Chen, J.L.-Y.; Scott, H.K.; Hesse, M.J.; Willis, C.L.; Aggarwal, V.K. Highly diastereo- and enantioselective allylboration of aldehydes using $\alpha$-substituted allyl/crotylpinacolboronic esters via in situ generated borinic esters. J. Am. Chem. Soc. 2013, 135, 5316-5319.

15. Hesse, M.J.; Essafi, S.; Watson, C.G.; Harvey, J.N.; Hirst, D.; Willis, C.L.; Aggarwal, V.K. Highly selective allylborations of aldehydes using $\alpha, \alpha$-disubstituted allylic pinacolboronic esters. Angew. Chem. Int. Ed. 2014, 53, 6145-6149. 
16. Pulis, A.P.; Fackler, P.; Aggarwal, V.K. Short stereoselective synthesis of the phytophthora universal mating hormone $\alpha 1$ using lithiation/borylation reactions. Angew. Chem. Int. Ed. 2014, 126, 4471-4474.

17. Roesner, S.; Brown, C.A.; Mohiti, M.M.; Pulis, A.P.; Rasappan, R.; Blair, B.J.; Essafi, S.; Leonori, D.; Aggarwal, V.K. Stereospecific conversion of alcohols into pinacolboronic esters using lithiation-borylation methodology with pinacolborane. Chem. Commun. 2014, 50, 4053-4055.

18. Pulis, A.P.; Blair, D.J.; Torres, E.; Aggarwal, V.K. Synthesis of enantioenriched tertiary boronic esters by the lithiation/borylation of secondary alkyl benzoates. J. Am. Chem. Soc. 2013, 135, 16054-16057.

19. Althaus M.; Mahmood, A.; Suárez, J.R.; Thomas, S.P.; Aggarwal, V.K. Application of the lithiation/borylation reaction to the preparation of enantioenriched allylic boron-reagents and subsequent in situ conversion into 1,2,4-trisubstituted homolallylic alcohols with complete control over all elements of stereochemistry. J. Am. Chem. Soc. 2010, 132, 4025-4028.

20. Mahmood, A.; Suárez, J.R.; Thomas, S.P.; Aggarwal, V.K. One-pot synthesis of 2,3,4,5,6pentasubstituted tetrahydropyrans using lithiation/borylation, allylation and Prins cyclisation reactions. Tetrahedron Lett. 2013, 54, 49-51.

21. Dearden, M.J.; Firkin, C.R.; Hermet, J.P.R.; O’Brien, P. A readily-accessible (+)-sparteine surrogate. J. Am. Chem. Soc. 2002, 124, 11870-11871.

22. Dixon, A.J.; McGrath, M.J.; O’Brien, P. Synthesis of (+)-(1R,2S,9S)-11-methyl-7,11diazatricyclo[7.3.1.0 $0^{2,7}$ ]tridecane, A (+)-sparteine surrogate. Org. Synth. 2006, 83, 141-144.

23. Hoppe, D.; Hense, T. Enantioselective synthesis with lithium/(-)-sparteine carbanion pairs. Angew. Chem. Int. Ed. 1997, 36, 2282-2316.

24. Gallagher, D.J.; Du, H.; Long, S.A.; Beak, P. Chiral organolithium complexes: The structure of $\beta$-lithiated $\beta$-phenylcarboxamides and the mechanism of asymmetric substitution in the presence of (-)-sparteine. J. Am. Chem. Soc. 1996, 118, 11391-11398.

25. Ebner, T.; Eichelbaum, M.; Fischer, P.; Meese, C.O. Stereospecific hydroxylation of (+)-sparteine in the rat. Arch. Pharm. 1989, 322, 399-403.

26. Richardson, T.I.; Rychnovsky, S.D. Filipin III: Configuration assignment and confirmation by synthetic correlation. J. Org. Chem. 1996, 61, 4219-4231.

27. Rychnovsky, S.D. Oxo polyene macrolide antibiotics. Chem. Rev. 1995, 95, 2021-2040.

28. Denmark, S.E.; Almstead, N.G. Allylation of carbonyls: Methodology and stereochemistry. In Modern Carbonyl Chemistry; Otera, J., Ed.; Wiley-VCH: Weinheim, Germany, 2000; Volume 10, p. 299.

29. Chemler, S.R.; Roush, W.R. Recent applications of the allylation reaction to the synthesis of natural products. In Modern Carbonyl Chemistry; Otera, J., Ed.; Wiley-VCH: Weinheim, Germany, 2000; Volume 11, p. 403.

30. Lachance, H.; Hall, D.G. Allylboration of carbonyl compounds. In Organic Reactions; Denmark, S.E., Ed.; John Wiley \& Sons: New York, NY, USA, 2008; Volume 73, p. 1.

31. Denmark, S.E.; Fu, J. Catalytic Enantioselective addition of allylic organometallic reagents to aldehydes and ketones. Chem. Rev. 2003, 103, 2763-2793. 
32. Roush, W.R. Methods of organic synthesis. In Stereoselective Synthesis; Helmchen, G., Hoffman, R.W., Muler, J., Schaumann, E., Eds.; Thieme Verlag: Stuttgart, Germany, 1996; Volume 3, p. 1410.

33. Hoffmann, R.W.; Zeiss, H.J. Diastereoselective synthesis of $\beta$-methyl homoallyl alcohols. Angew. Chem. Int. Ed. 1979, 18, 306-309.

34. Brown, H.C.; Bhat, K.S. Enantiomeric Z- and E-crotyldiisopinocampheylboranes. Synthesis in high optical purity of all four possible stereoisomers of $\beta$-methylhomoallyl alcohols. J. Am. Chem. Soc. 1986, 108, 293-294.

35. Rauniyar, V.; Hall, D.G. Rationally improved chiral Brønsted acid for catalytic enantioselective allylboration of aldehydes with an expanded reagent scope. J. Org. Chem. 2009, 74, 4236-4241.

36. Penner, M.; Rauniyar, V.; Kaspar, L.T.; Hall, D.G. Catalytic asymmetric synthesis of palmerolide via organoboron methodology. J. Am. Chem. Soc. 2009, 131, 14216-14217.

37. Chen, M.; Handa, M.; Roush, W.R. Enantioselective synthesis of 2-methyl-1,2-syn- and 2-methyl1,2-anti-3-butenediols via allene hydroboration-aldehyde allylboration reaction sequences. J. Am. Chem. Soc. 2009, 131, 14602-14606.

38. Carosi, L.; Hall, D.G. Catalytic enantioselective preparation of alpha-substituted allylboronates. One-pot addition to functionalized aldehydes and a route to chiral allylic trifluoroborate salts. Angew. Chem. Int. Ed. 2007, 46, 5913-5918.

39. Kister, J.; DeBaillie, A.C.; Lira, R.; Roush, W.R. Stereoselective synthesis of $\gamma$-substituted (Z)-allylic boranes via kinetically controlled hydroboration of allenes with 10-TMS-9-borabicyclo[3.3.2]decane. J. Am. Chem. Soc. 2009, 131, 14174-14178.

40. González, A.Z.; Román, J.G.; Alicea, E.; Canales, E.; Soderquist, J.A. (E)-2-Boryl-1,3-butadiene derivatives of the 10-TMS-9-BBDs: Highly selective reagents for the asymmetric synthesis of anti-1,2-disubstituted-3,4-pentadien-1-ols. J. Am. Chem. Soc.2009, 131, 1269-1273.

41. Corey, E.J.; Rhodes, J.J. The application of the formyl hydrogen bond postulate to the understanding of enantioselective reactions involving chiral boron lewis acids and aldehydes. Tetrahedron Lett.1997, 38, 37-40.

42. Yamamoto, Y.; Fjikawa, R.; Yamada, A.; Miyaura, N. Synthesis of B-trisubstituted borazines via the rhodium-catalyzed hydroboration of alkenes with $N, N^{\prime}, N^{\prime \prime}$-trimethyl or $N, N^{\prime}, N^{\prime \prime}$-triethylborazine, Chem. Lett.1999, 1069-1071.

43. Andersen, M.W.; Hildebrndt, B.; Kosher, G.; Hoffmann, R.W. Stereoselective synthesis of alcohols, XXX: $E$ - and Z-pentenylboronates, reagents for simple diastereoselection on addition to aldehydes. Chem. Ber. 1989, 122, 1777-1781.

44. Hoffmann, R.W.; Dirich, K.; Kosher, G.; Strürmer, R. Stereoselective synthesis of alcohols, XXXI: Stereoselective C-C bond formation using chiral Z-pentenylboronates. Chem. Ber. 1989, 122, 1783-1786.

45. Fang, G.Y.; Aggarwal, V.K. Asymmetric synthesis of $\alpha$-substituted allylic boranes and their application in synthesis of iso-agatharesinol. Angew. Chem. Int. Ed. 2007, 46, 359-362.

46. Howells, D.; Robiette, R.; Fang, G.Y.; Knowles, L.S.; Woodrow, M.D.; Harvey, J.N.; Aggarwal, V.K. Reactions of silyl-stabilised sulfur ylides with organoboranes: Enantioselectivity, mechanism, and understanding. Org. Biomol. Chem. 2008, 6, 1185-1189. 
47. Soderquist, J.A.; Martinez, J.; Oyola, Y.; Dock, I. Novel route of carboxylic acids via the DCME reaction. Tetrahedron Lett. 2004, 45, 5541-5545.

48. Brown, H.C.; Kim, K.W.; Cole, T.A.; Singaram, B. Chiral synthesis via organoboranes. 8. Synthetic utility of boronic esters of essentially $100 \%$ optical purity. Synthesis of primary amines of very high enantiomeric purities. J. Am. Chem. Soc. 1986, 108, 6761-6764.

Sample Availability: Samples of the compounds $(\mathbf{8 I}-\mathbf{8 V})$ are available from the authors.

(C) 2015 by the authors; licensee MDPI, Basel, Switzerland. This article is an open access article distributed under the terms and conditions of the Creative Commons Attribution license (http://creativecommons.org/licenses/by/4.0/). 